\title{
A MUSE map of the central Orion Nebula (M 42)
}

\author{
Peter M. Weilbacher ${ }^{1}$, Ana Monreal-Ibero ${ }^{2}$, Wolfram Kollatschny ${ }^{3}$, Adam Ginsburg ${ }^{4}$, Anna F. McLeod ${ }^{4}$, \\ Sebastian Kamann ${ }^{3}$, Christer Sandin ${ }^{1}$, Ralf Palsa ${ }^{4}$, Lutz Wisotzki ${ }^{1}$, Roland Bacon ${ }^{7}$, Fernando Selman ${ }^{8}$, \\ Jarle Brinchmann ${ }^{5}$, Joseph Caruana ${ }^{1}$, Andreas Kelz ${ }^{1}$, Thomas Martinsson ${ }^{5,9,10}$, \\ Arlette Pécontal-Rousset ${ }^{7}$, Johan Richard ${ }^{7}$, and Martin Wendt ${ }^{1,6}$ \\ ${ }^{1}$ Leibniz-Institut für Astrophysik Potsdam (AIP), An der Sternwarte 16, 14482 Potsdam, Germany \\ e-mail: pweilbacher@aip.de \\ 2 GEPI, Observatoire de Paris, CNRS, Université Paris-Diderot, Place Jules Janssen, 92190 Meudon, France \\ 3 Institut für Astrophysik, Universität Göttingen, Friedrich-Hund-Platz 1, 37077 Göttingen, Germany \\ ${ }^{4}$ ESO, European Southern Observatory, Karl-Schwarzschild Str. 2, 85748 Garching bei München, Germany \\ 5 Leiden Observatory, Leiden University, PO Box 9513, 2300 RA Leiden, The Netherlands \\ 6 Institut für Physik und Astronomie, Universität Potsdam, 14476 Golm, Germany \\ 7 CRAL, Observatoire de Lyon, CNRS, Université Lyon 1, 9 avenue Charles André, 69561 Saint-Genis Laval Cedex, France \\ 8 ESO, European Southern Observatory, 3107 Alonso de Córdova, Santiago, Chile \\ 9 Instituto de Astrofísica de Canarias (IAC), 38205 La Laguna, Tenerife, Spain \\ 10 Departamento de Astrofísica, Universidad de La Laguna, 38206 La Laguna, Tenerife, Spain
}

Received 13 May 2015 / Accepted 16 August 2015

\begin{abstract}
We present a new integral field spectroscopic dataset of the central part of the Orion Nebula (M 42), observed with the MUSE instrument at the ESO VLT. We reduced the data with the public MUSE pipeline. The output products are two FITS cubes with a spatial size of $\sim 5.9 \times 44^{\prime} 9$ (corresponding to $\sim 0.76 \times 0.63 \mathrm{pc}^{2}$ ) and a contiguous wavelength coverage of $4595 \ldots 9366 \AA$, spatially sampled at 0.2 . We provide two versions with a sampling of $1.25 \AA$ and $0.85 \AA$ in dispersion direction. Together with variance cubes these files have a size of 75 and $110 \mathrm{GiB}$ on disk. They are the largest integral field mosaics to date in terms of information content. We make them available for use in the community. To validate this dataset, we compare world coordinates, reconstructed magnitudes, velocities, and absolute and relative emission line fluxes to the literature values and find excellent agreement. We derive a 2D map of extinction and present de-reddened flux maps of several individual emission lines and of diagnostic line ratios. We estimate physical properties of the Orion Nebula, using the emission line ratios [N II] and [S III] (for the electron temperature $T_{\mathrm{e}}$ ) and [S II] and [Cl III] (for the electron density $N_{\mathrm{e}}$ ), and show 2D images of the velocity measured from several bright emission lines.
\end{abstract}

Key words. H II regions - ISM: individual objects: M 42 - open clusters and associations: individual: Trapezium cluster

\section{Introduction}

An H II region is a diffuse nebula whose gas is heated and ionized by the ultraviolet radiation of early-type massive stars (see Shields 1990; Osterbrock \& Ferland 2005). H II regions are typically found in the arms of spiral galaxies and/or irregular galaxies and present spectra with strong emission lines visible even at cosmological distances. Galactic H II regions, in particular, can be seen as small-scale versions of the extreme events of star formation occurring in starburst galaxies (e.g., Weilbacher et al. 2003; Alonso-Herrero et al. 2009; García-Marín et al. 2009; Cairós et al. 2015). As such, they are laboratories that offer an invaluable opportunity to study the interplay between recent and/or ongoing star formation - in particular massive stars - and their surrounding interstellar medium, including gas and dust, at a high level of detail.

One of the best-studied Galactic H II regions (and the closest) is the Orion Nebula (M 42), which is visible to the naked eye. It is often one of the first objects targeted with a new instrument,

\footnotetext{
* Data products are available at http://muse-vlt.eu/science

$\star \star$ Based on observations made with ESO telescopes at the La Silla Paranal Observatory under program ID 60.A-9100(A).
}

first, to see if something new can be discovered and, second, to use the plethora of existing observations for comparison to validate a new system. A review of the nebula and its stellar content can be found in O'Dell (2001). Spectroscopic studies of the ionized gas, confined to one or several slit positions, have partially characterized Orion's emission spectrum (e.g., Baldwin et al. 1991; Pogge et al. 1992; Osterbrock et al. 1992; Mesa-Delgado et al. 2008; O’Dell \& Harris 2010).

However, H II regions are rarely as simple as the textbooklike Strömgren (1939) spheres, and Orion is no exception. Indeed, M 42 is thought to be only a thin blister of ionized gas at the near side of a giant molecular cloud (Zuckerman 1973; Israel 1978; van der Werf et al. 2013). To make most of the opportunity to observe an H II region at the level of detail offered by Orion, spatially resolved maps with high-quality spectral information in terms of depth, spatial, and spectral resolution are needed.

The most efficient way to gather this information nowadays is probably the use of Integral Field Spectroscopy. Sánchez et al. (2007) released a first dataset based on this technique to the community (using the PPak mode of PMAS, Kelz et al. 2006), mapping most of the Huygens region - the central part of the nebula with the highest surface brightness. However, the data were not taken under ideal weather conditions and so were 
poorly flux-calibrated. They were shallow due to very short exposure time and of low spatial and spectral resolution. Some of these aspects (depth and spectral resolution) have been improved in a new mosaic mapping a similar area (Núñez-Díaz et al. 2013). However, the spatial resolution of these data are still relatively low. At the moment, however, this improved dataset is not publicly available in reduced form. Additionally, there are several studies with very good data quality in terms of depth, spectral, and spatial resolution devoted to the study of invidiual targets within the Orion Nebula that observed the interplay of gas and stars in proplyds and Herbig-Haro objects (e.g., Vasconcelos et al. 2005; Mesa-Delgado et al. 2011, 2012; Tsamis \& Walsh 2011; Tsamis et al. 2013; Núñez-Díaz et al. 2012). Therefore, they only mapped very small $\left(\sim 10^{\prime \prime}\right)$ rectangular areas. None of these currently existing datasets satisfy all of the following requirements: i) a large mapped area; ii) depth; iii) ample spectral coverage; and iv) good spatial and spectral resolution.

Here, we present what we call true imaging spectroscopy of the Huygens region of the Orion Nebula, observed with the MUSE integral field spectrograph mounted on VLT UT4 "Yepun". MUSE comes close to producing the "perfect dataset" mentioned by O'Dell (2001): it samples the Huygens region with high spatial sampling $(0.2)$ and reasonable spectral resolution $(R \sim 3000)$, and covers a large dynamic range.

There are two aims in this work. On the one hand, from the technical point of view, this is one of the first sets of MUSE data and as such, it was taken with the main goal of testing offsets larger than the field of view and stress-testing the data flow system related to the new instrument. On the other hand, from a scientific point of view, given the lack of a high-quality and science-ready set of spectrophotometric data of the whole Huygens region, we wanted to provide the community with such data.

In this paper, we describe the observations and the data reduction (Sect. 2), validate the new data against literature values (Sect. 3), describe a few unusual artifacts in the MUSE dataset (Sect. 4), and demonstrate how the MUSE datacube can be used for an analysis of both atomic and ionized gas (Sect. 5), before we conclude with a few general remarks (Sect. 6).

We assume a distance of $D=440$ pc (O'Dell \& Henney 2008) for the Orion nebula. This implies a linear scale of $0.0021 \mathrm{pc} \mathrm{arcsec}^{-1}$. The field of view of the MUSE dataset then corresponds to $\sim 0.76 \times 0.63 \mathrm{pc}^{2}$.

\section{Observations and data reduction}

M 42 was observed as part of the first commissioning run (Bacon et al. 2014) of the MUSE instrument on the VLT. After some test exposures during the night before the actual observations, a uniform exposure time of $5 \mathrm{~s}$ per exposure was chosen as a compromise so as not to saturate the bright emission lines but to give sufficient signal-to-noise ratio $(\mathrm{S} / \mathrm{N})$ in the outer regions. On 2014 February 16 between 01:02:59 and 03:34:31 (UTC), 60 exposures over a 6-by-5 mosaic were taken. Two exposures per position were observed, with the same center but alternating position angles of 0 and $90 \mathrm{deg}$. The positions of the exposures are shown schematically in Fig. 1. To be able to create a contiguous grid, the positions were offset by $58^{\prime \prime}$, which is somewhat smaller than the MUSE FOV.

Observation of the standard star GD 71 at 00:15:14 UTC and an airmass of 1.33 allowed a spectrophotometric calibration of the data. No sky exposures were taken. Daytime calibrations of the morning after the observing night were used.

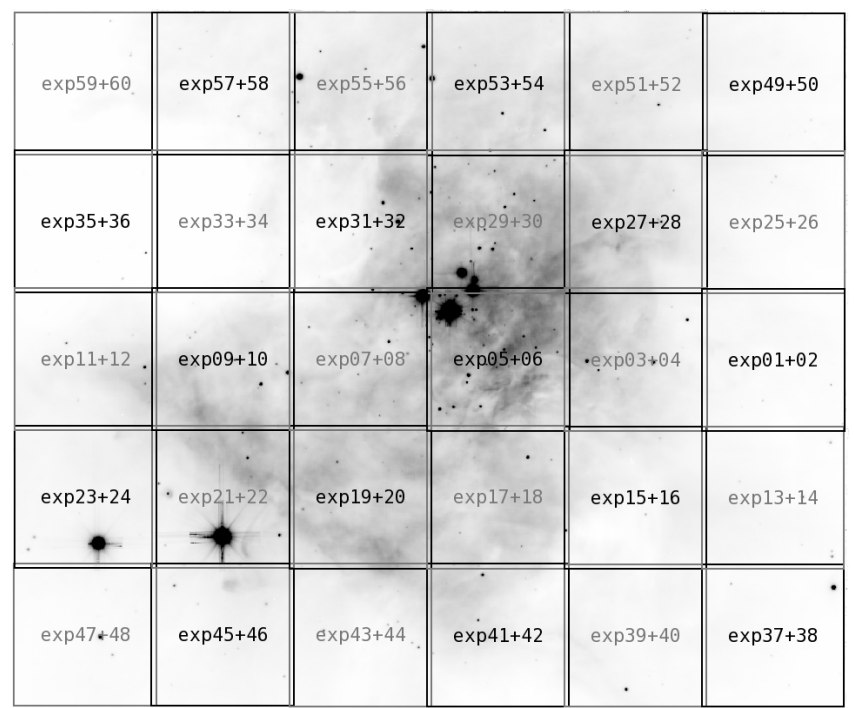

Fig. 1. Inverse grayscale representation of the white-light image of the final mosaic. The positions of the observations are marked and annotated with the exposure numbers in the sequence from 1 to 60 . Each box represents the approximate field of view of a single MUSE exposure, about $1^{\prime} \times 1^{\prime}$. The full field covered is $\sim 5^{\prime} .9 \times 4^{\prime} .9$, centered on $\alpha=5^{\mathrm{h}} 35^{\mathrm{m}} 17^{\mathrm{s}} .0, \delta=-5^{\circ} 23^{\prime} 43^{\prime \prime}$, with north to the top and east to the left.

The observing conditions were good, with photometric sky and DIMM seeing varying between $0 .{ }^{\prime} 67$ and 1'.25. The M 42 mosaic was observed after transit, with airmass values ranging from 1.067 to 1.483 . During the observations, the moon had an illumination of $95 \%$, a distance of $\sim 87^{\circ}$ from the target, and rose from $10^{\circ}$ to $45^{\circ}$ in elevation.

The reduction used the dedicated MUSE pipeline (Weilbacher et al. 2012, and in prep.) through the EsoREX program. We used a development version of the pipeline, but the code was very close to the 1.0 release $^{1}$ (patches are available on request).

For the basic calibration we followed the standard procedure to reduce MUSE data: combine ten bias images to form a master bias, combine five lamp-flat exposures, and use one exposure of each arc lamp to derive the wavelength solution. Eleven skyflats, taken during the evening twilight preceding the science exposures, were combined and used to create a $3 \mathrm{D}$ correction of the illumination in the range $\lambda=5000 \ldots 8000 \AA^{2}$.

The geometry of the instrument was derived from a calibration sequence taken on 2014-02-05, the astrometric solution of the MUSE field of view was computed from an observation of a field in NGC 3201 on 2014-02-09. These calibrations were found to be valid for the full period of the first commissioning run of the instrument and were also shipped with the MUSE pipeline.

We then applied all calibrations to both the standard star exposure and all 60 science exposures, making use of a table of additional bad pixels of the CCDs that was created after the completion of MUSE commissioning runs, and which is shipped with the pipeline. For datasets with longer exposure times and lower contrast, the pipeline usually manages to correct

1 Available from ESO via http://www.eso.org/sci/software/ pipelines/muse/muse-pipe-recipes.html

2 Redder wavelengths were excluded from the correction because the second order in the extended mode of MUSE created extra artifacts beyond $\sim 8100 \AA$. 
the zero-order of the dispersion solution on a per-IFU ${ }^{3}$ basis using Gaussian fits as centroiding of bright sky emission lines. In the case of the M42 mosaic, extreme contrast differences in some exposures and IFUs and the low sky emission background made this process unreliable. We therefore used the following procedure: we assume that the line [O I] 5577 is dominated by telluric emission (see, e.g., Baldwin et al. 2000), so it was taken as baseline reference for each exposure and IFU. Since the shifts on the CCD are were likely to change smoothly during the time of our observation, we iteratively fitted a linear relation to the MJD-OBS against the pipeline-computed wavelength offset, separately for each IFU. Deviant shifts were aggressively purged at the $2 \sigma$ level. The wavelength zeropoint was then reset according to this linear relation with time.

Since the observations were done in extended mode, which incurs a second-order overlap at the red end of the wavelength coverage, the creation of the flux response curve needed extra care. We ran the pipeline recipe (muse_standard) with both circular flux integration and flux integration using Moffat profile fits. Circular apertures were used for wavelengths below $8334 \AA$, as they integrate slightly more flux than the Moffat fits and create a smoother response curve. Beyond $8430 \AA$, the circular aperture also integrates significant flux from the diffuse second-order, and the Moffat fit is a better representation of the total flux in the 1 st order. At the transition wavelength, both curves have approximately the same slope and the curve from the Moffat fit was shifted slightly to account for the offset of both curves at this wavelength. The resulting merged response curve was again applied to the data of the standard star. A comparison of the reference spectrum with a spectrum extracted from that calibrated cube showed deviations typically below $5 \%$.

The merged response and the astrometric calibration were then applied to each exposure individually. Creating and applying the response function used the average atmospheric extinction curve by Patat et al. (2011) as shipped with the MUSE pipeline. We let the pipeline automatically correct the atmospheric refraction (with the default method using the formula of Filippenko 1982) and the barycentric velocity offset, but did not attempt to remove the sky background or the telluric absorption in the data ${ }^{4}$. No attempts were made to homogenize the seeing along the wavelength direction, or between the 60 exposures.

All exposures were combined into a single cube. Since the observation strategy included rotation, the data were affected by the derotator wobble ${ }^{5}$ so that each exposure had to be repositioned slightly. Luckily, each pointing contained at least one star that was also listed in the 2MASS catalog (Skrutskie et al. 2006), so we used the 2MASS positions as references for the offsets that were applied when reconstructing the full cube. The effect is that the absolute astrometry of the final cube is tied to the 2MASS coordinates, similar to the HST mosaic of the Orion Nebula (Robberto et al. 2013).

We created a first full cube with the standard pipeline sampling of $0.2 \times 0.2 \times 1.25 \AA$. However, as discussed below, we

\footnotetext{
3 IFU = Integral Field Unit; for MUSE this is one of the 24 subunits consisting of image slicer, spectrograph, and $\mathrm{CCD}$, each covering about $60^{\prime \prime} \times 22^{\prime \prime} 5$ on the sky.

${ }^{4}$ It should be possible to use tools that rely on atmospheric modeling instead of dedicated calibration exposures to subtract the sky background and remove the telluric features once spectra are extracted from the cube. Examples of such tools are SKYCORR (Noll et al. 2014a,b) and MOLECFIT (Smette et al. 2015b,a).

5 This "wobble" refers to a decentering of the optical axis of the MUSE derotator with the axis of the VLT.
}

Table 1. FITS extensions in the provided files ${ }^{a}$.

\begin{tabular}{lll}
\hline \hline EXTNAME & $\lambda$-range $[\AA]$ & Comment \\
\hline DATA & $4595.00 \ldots 9366.05$ & data values \\
STAT & $4595.00 \ldots 9366.05$ & data variance \\
\hline white & $4650.00 \ldots 9300.00$ & \\
Johnson_V & & $V$-band filter \\
Cousins_R & & $R$-band filter \\
Cousins_I & & $I$-band filter \\
\hline Halpha & $6556.78 \ldots 6568.78$ & H $\alpha$ \\
NII_both & $6542.06 \ldots 6554.06$, & both [N II] lines... \\
& $6577.39 \ldots 6589.39$ & $\ldots(6548$ and 6584) \\
Halpha_NII_OFF & $6533.05 \ldots 6538.05$, & off-band for H $\alpha \ldots$ \\
& $6593.40 \ldots 6598.40$ & $\ldots$ and [N II] \\
OIII_both & $4953.92 \ldots 4965.92$, & both [O III] lines... \\
& $5000.85 \ldots 5012.85$ & $\ldots(4959$ and 5007) \\
OIII_OFF & $4969.92 \ldots 4996.85$ & off-band for [O III] \\
Hbeta & $4855.32 \ldots 4867.32$ & H $\beta$ \\
Hbeta_OFF & $4846.32 \ldots 4851.31$, & off-band... \\
& $4871.33 \ldots 4876.32$ & $\ldots$ for H $\beta$ \\
\hline
\end{tabular}

Notes. ${ }^{(a)}$ The upper part of the table contains both data cubes, the middle part the images from standard pipeline filters, and the bottom images from specially created filters.

then chose a higher wavelength sampling for the final cube to partially overcome the undersampling of MUSE data in the dispersion direction ${ }^{6}$. Hence, a second cube was reconstructed with a sampling of $0.2 \times 0$. spaxel $^{-1}$ in spatial and a linear step of $0.85 \AA_{\text {A pixel }}^{-1}$ in wavelength direction. We call this cube the HR $\mid 0.85$ cube, while the cube with the standard sampling is the LR 1.25 cube. Both cubes have approximately the same wavelength coverage of $4595 \ldots 9366 \AA$. The extent on the sky is exactly the same, 5 '. $88 \times 44^{\prime} .92$, but patches up to about 10 spaxels at the edge are not covered by data and filled with NaN values. The total size of the cubes is $1766 \times 1476 \times 3818$ voxels for LR 1.25 and $1766 \times 1476 \times 5614$ for HR $\mid 0.85$ and they are stored in units of $10^{-20} \mathrm{erg} \mathrm{s}^{-1} \mathrm{~cm}^{-2} \AA^{-1}$ in the DATA extension of the FITS file. The MUSE pipeline also reconstructs a variance $\left(\sigma^{2}\right)$ cube and stores it in the STAT extension in the same file. Several image extensions are available as well, averaging the cube either using known filter functions or using constant weights across interesting wavelength ranges around some lines (see Table 1 for details). These image extensions were created to be used only to locate interesting features in the cube, not for scientific analysis. The file size of the full dataset is $75 \mathrm{GiB}$ (LR $\mid 1.25)$ and $110 \mathrm{GiB}(\mathrm{HR} \mid 0.85)$.

For the purpose of the demonstration in this paper, we finally decided to use the LR $\mid 1.25$ data for everything except the spatially resolved velocity analysis of the ionized gas, where $\mathrm{HR} \mid$ 0.85 gives much lower systematic structures (see Sect. 3.4). However, the spatial calibration and the spectrophotometric accuracy is exactly the same for the HR $\mid 0.85$ data, so the values quoted for the data quality in Sects. 3.1, 3.3, 3.5, and 3.6 refer to both datasets.

\section{Fidelity of the data}

Since MUSE is a new instrument and the data reduction software is new, we have to carefully check the fidelity of the data to ensure its scientific usefulness.

6 In this dataset, however, this choice leads to other artifacts, see Sect. 4 and Fig. 9. 


\subsection{Accuracy of the coordinate system}

To verify the accuracy of the world coordinate system (WCS) in the MUSE cube, we determine positions in the reconstructed image integrated over the Johnson $V$ filter (extension Johnson_V in the FITS file). Applying DAOFIND in $\operatorname{IRAF}^{7}$ to this image yields 259 detections, some of which are spurious sources.

Matching the list of point sources detected in MUSE to the 2MASS catalog (Skrutskie et al. 2006) results in 96 matches closer than $1^{\prime \prime}$. After removing spurious sources, undetected double stars (listed as sources in the 2MASS catalog), and stars saturated in the MUSE cube, we are left with 90 matched sources. Their separations are 0.'108 \pm 0.072 (mean and standard deviation, or 0.'097 \pm 0. .'045 using median and median absolute deviation). Using the same procedure, but matching MUSE detections against the HST ACS catalog of the Orion Nebula cluster as given by Robberto et al. (2013), we find 83 valid matches, giving an overall agreement of $0{ }^{\prime} 163 \pm 0.078$ (mean and standard deviation). This is in line with the accuracy of the HST catalog matched against 2MASS point sources (max. allowed separation $0 . ' 5$, resulting in $0{ }^{\prime} 138 \pm 0.085$ ) and comparable to the astrometric accuracy of the 2MASS point source catalog itself, $\lesssim 100$ mas given in Skrutskie et al. (2006).

\subsection{Quality of the atmospheric refraction correction}

Since the atmospheric refraction present in the raw MUSE data was corrected by the pipeline reduction, compact sources in the field do not show strong gradients across several spatial pixels. Nevertheless, the formula to compute the refractive index of air (taken from Filippenko 1982) is imperfect, so some residuals are left in the data.

We test the residuals using the centers of four bright stars in the field. The centers of the stars were measured in each wavelength plane of the final cube by two methods: 1) by fitting a Moffat function; and 2) by computing the marginal centroid as with IMCENTROID in IRAF. The results are shown in Fig. 2.

The strongest deviations from the mean centroid position of each star occur in regions of high background (strong nebular emission lines) and low throughput (telluric absorption). Ignoring these wavelength ranges, the typical deviations from the mean centroid position are smaller than $0 . ' 05$ or one-fourth of a spatial element of the cube.

\subsection{Magnitudes and colors}

We used the same stellar spectra already extracted using the Moffat fit in Sect. 3.2 to determine how well we can reproduce stellar magnitudes using the MUSE data.

The extracted spectra are shown in Fig. 3. The telluric absorption that is visible does not significantly affect measurement of the integrated magnitudes ( $R$-band: $0.007 \mathrm{mag}, I$-band: $0.023 \mathrm{mag}$ ). Nevertheless, for this test, we replaced the absorbed regions in the spectrum with an average value before integrating the spectra over the filter functions (we used Johnson $V$, and Cousins $R$ and $I$ ).

The effect of nebular line emission that was not optimally subtracted by the Moffat fit is less certain. Indeed, LL Ori shows intrinsic Balmer and CaII-triplet emission that cannot be 7 IRAF is written and supported by the National Optical Astronomy
Observatories (NOAO) in Tucson, Arizona. NOAO is operated by the
Association of Universities for Research in Astronomy (AURA), Inc., under cooperative agreement with the National Science Foundation.

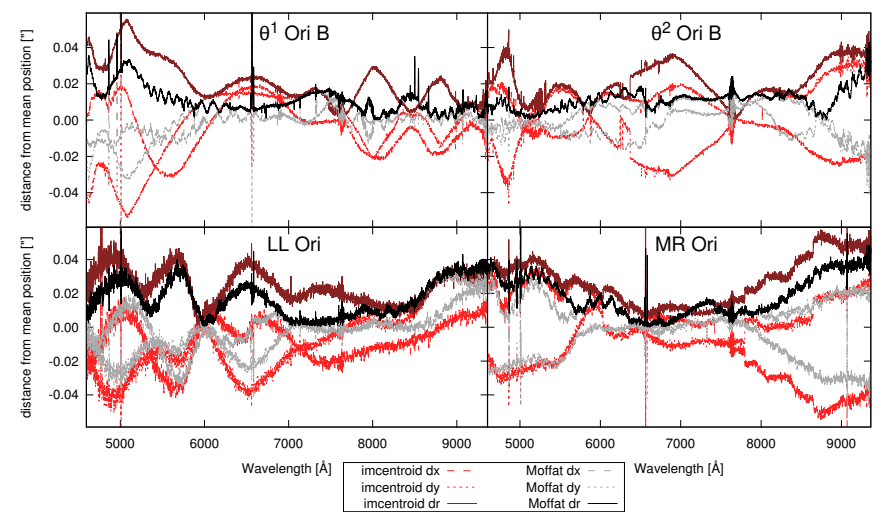

Fig. 2. Centroiding residuals versus wavelength for four bright stars in the field. Each panel shows horizontal and vertical distances to the mean center of the star in lighter dashed and dotted curves, and the radius as a solid dark curve. Red curves are for IMCENTROID measurements, black lines for Moffat fits.

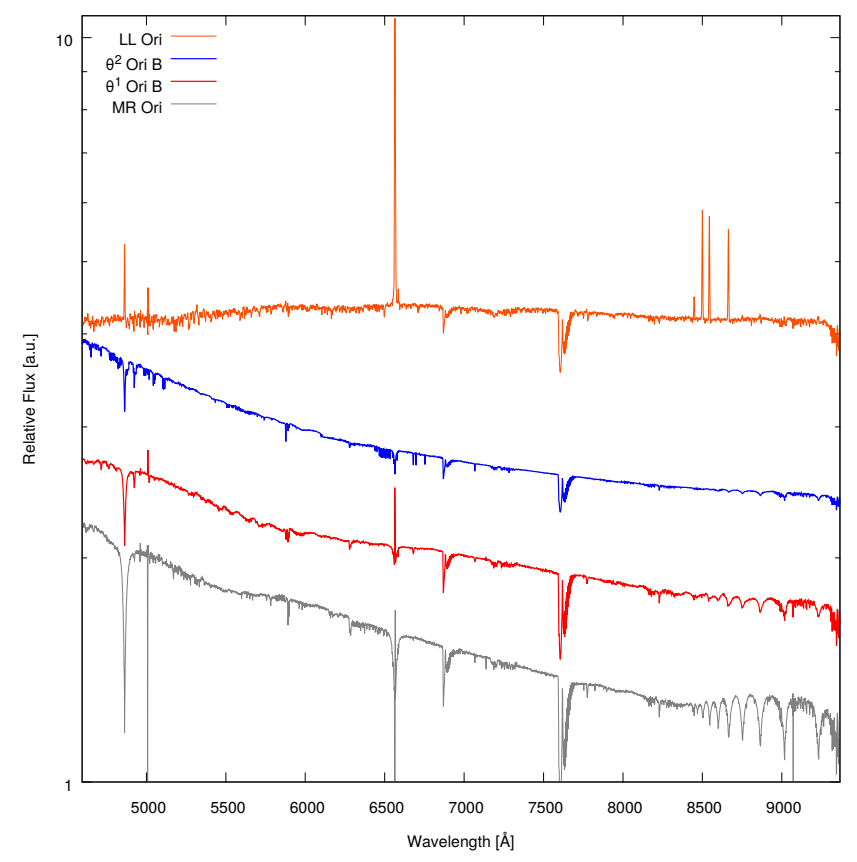

Fig. 3. Extracted spectra of four bright stars in the field; the flux is displayed in arbitrary logarithmic units, the individual spectra are scaled and offset for better visibility, and not in order of stellar magnitude.

explained by residuals of nebular emission (see also Hillenbrand 1997), whereas small residuals of [O III] 5007 and [N II] 6584 are very likely of nebular origin. Since the equivalent widths of the emission features are low $-E W(\mathrm{H} \alpha) \approx-42.5 \AA$ for LL Ori - the influence on the broadband magnitudes is rather low. An agreement of a few hundredths of a magnitude in the $V$-band can be regarded as very satisfactory. The $V$-band differences quoted in Table 2 correspond to flux differences of up to $2.7 \%$.

However, the comparison with $R$ and $I$ filters, where available, remains puzzling. Only the $I$-band magnitudes given in the table of Hillenbrand (1997) are close to our measurements, and these are only available for two of our four stars used for this experiment. As we have argued in Sect. 2 and checked in a different way in Sect. 3.6, differences of 0.1 to 0.18 mag or up to $18 \%$ in flux (as seen relative to the measurements of Ducati 2002; Da Rio et al. 2009) are unlikely to be a problem with the relative 
Table 2. Comparison of integrated magnitudes to the literature.

\begin{tabular}{lcrc}
\hline \hline Object & Band & $m_{\text {MUSE }}[\mathrm{mag}]$ & $m_{\text {lit }}-m_{\text {MUSE }}[\mathrm{mag}]$ \\
\hline$\theta^{1}$ Ori B & $V$ & 7.990 & $-0.03^{a}$ \\
& $R$ & 7.760 & \\
& $I$ & 7.422 & \\
$\theta^{2}$ Ori B & $V$ & 6.389 & $0.009^{a}, 0.021^{d}$ \\
& $R$ & 6.403 & $-0.104^{a}$ \\
& $I$ & 6.356 & $\left(4.694^{b, c}\right), 0.054^{d}$ \\
LL Ori & $V$ & 11.499 & $0.018^{b}, 0.021^{d}$ \\
& $R$ & 10.835 & \\
MR Ori & $I$ & 10.218 & $-0.184^{b},-0.038^{d}$ \\
& $V$ & 10.551 & $0.027^{b}$ \\
& $R$ & 10.286 & \\
& $I$ & 10.010 & $-0.174^{b}$ \\
\hline
\end{tabular}

Notes. ${ }^{(a)}$ Compared to magnitude given by Ducati (2002); ${ }^{(b)}$ compared to magnitude given by Da Rio et al. $(2009) ;{ }^{(c)}$ the value given by $\mathrm{Da}$ Rio et al. (2009) for $\theta^{2}$ Ori B $\left(m_{I}=11.050 \mathrm{mag}\right)$ is clearly faulty; (d) compared to magnitudes given by Hillenbrand (1997).

flux calibration of the MUSE spectra, which is accurate to at least $5 \%$.

To further investigate the difference, we also convolved our spectra with the filter plus CCD throughputs of the ESO WFI setup used by Da Rio et al. (2009). This made the agreement even worse. Since all stars in our field of view are likely variable at some level - of the four stars we analyzed here, all except $\theta^{2}$ Ori B are listed in the variable star catalog of (Samus et al. 2009) - one should not expect high precision of the comparison, but as variability likely affects observations in different filters in a similar way, this cannot explain the differences we see here. We therefore have to assume that the zeropoints applied by Da Rio et al. include an unknown component that causes a shift of the central effective wavelength of the red filters, but less of a shift for the green filter ${ }^{8}$. Since the Orion Nebula is too bright for SDSS stellar photometry to work and all four stars are marked as saturated in the HST ACS catalog of Robberto et al. (2013), we cannot cross-check our reconstructed magnitudes with a betterstudied photometric system.

\subsection{Derived velocities}

MUSE has a moderate velocity resolution (about $107 \mathrm{~km} \mathrm{~s}^{-1}$ at $7000 \AA$ ), and the line spread function is slightly undersampled $^{9}$. As a consequence it is challenging to measure accurate velocity centroids for single narrow spectral features such as emission lines in HII regions. Nevertheless, we compare our derived velocities against the values given by Baldwin et al. (2000, hereafter B00).

The MUSE cube is corrected to barycentric velocities ${ }^{10}$, so we can directly check our velocities against their heliocentric reference value of $+11.9 \mathrm{~km} \mathrm{~s}^{-1}$. If we extract a spectrum over the same aperture as the "blue" slit of B00, and fit all bright and a few fainter emission lines with Gaussian profiles, we can

\footnotetext{
8 This could be due to the relative throughput of atmosphere or telescope that are unknown to us, see, e.g., Doi et al. (2010) for details on filter profile determinations and the effect of the atmosphere.

9 MUSE has a typical FWHM of $2.5 \AA$ sampled at about $1.25 \AA$ pixel $^{-1}$.

10 The difference between barycentric and heliocentric velocities at the time of observations was less than $10 \mathrm{~m} \mathrm{~s}^{-1}$.
}

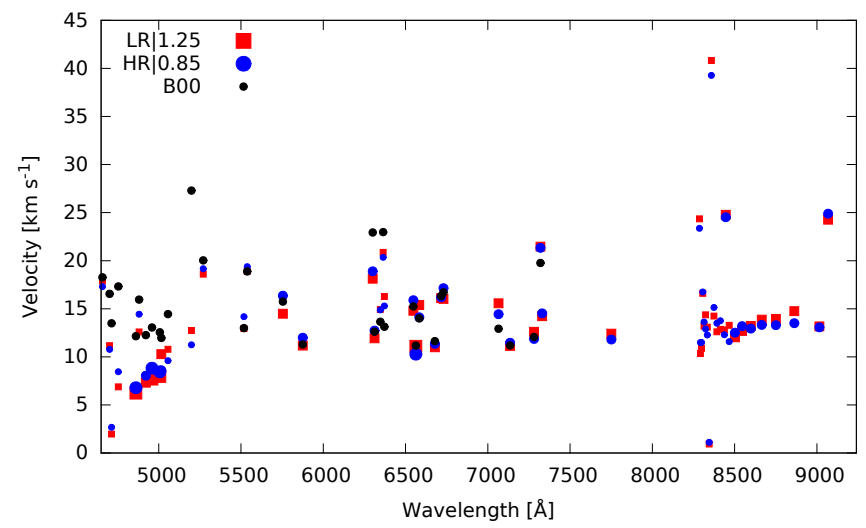

Fig. 4. Velocities measured within the "blue" slit of Baldwin et al. (2000, B00). These are corrected to barycentric velocity for our data (red squares and blue circles) and to heliocentric for B00 (black circles). The point size of our measurements changes with the relative flux of the lines, such that the brightest lines have the largest symbols.

plot the resulting velocities for both of our cubes (LR $\mid 1.25$ and $\mathrm{HR} \mid 0.85)$ as well as the reference values from B00.

In Fig. 4 we show the result. We can reproduce the average velocity of about $12 \mathrm{~km} \mathrm{~s}^{-1}$ reported by $\mathrm{B} 00^{11}$. This is seen for most of the Paschen lines in the very red (that were not measured in the B00 setup) and - with some scatter - for most other lines in the wavelength range $5250<\lambda<8000 \AA$. The bright lines below $5250 \AA$, however, show a deviation from this mean velocity, in the sense that we measure velocities about $6 \mathrm{~km} \mathrm{~s}^{-1}$ below those derived by B00. While the fainter lines at similar wavelengths do not all show this offset, they are partly blended with neighboring lines, and are therefore less trustworthy.

Several strong outliers are visible at the red end of the spectrum in Fig. 4. These are three of the fainter Paschen lines (Pa22, $\mathrm{Pa} 23$, and Pa30) and are most likely due to blending with another unidentified weak line. However, the strong and relatively isolated lines OI 8446 and [S III] 9069 also show a strong offset of $\sim 12 \mathrm{~km} \mathrm{~s}^{-1}$. Since the surrounding Paschen lines follow the normal trend very well, this casts doubts on the reliability of the reference wavelengths (we used 8446.462 and 9068.6 from the NIST database; Kramida et al. 2014). Indeed, taking the reference value of $9068.9 \AA$ as quoted by Osterbrock et al. (1992) for [S III], we find a velocity of 14.35 and $14.97 \mathrm{~km} \mathrm{~s}^{-1}$ for our LR|1.25 and HR|0.85 data, respectively, perfectly in line with the general trend.

We therefore conclude that in the range $\lambda<5250 \AA$ the MUSE data likely shows a problem with the wavelength calibration, on the level of up to $0.1 \AA$ (less than one-tenth of a MUSE pixel), while no systematic deviations larger than $\sim 3 \mathrm{~km} \mathrm{~s}^{-1}$ were found for wavelengths $\lambda>5250 \AA$.

We then compare maps of emission line velocities over the full field. These velocities were derived using single Gaussian profile fits to individual lines, and the maps show systematic patterns. These systematics are much more pronounced in the LR $\mid 1.25$ cube than in the HR|0.85 data. This is demonstrated in Fig. 5, which shows the 2D velocity field recovered from Gaussian centroids of the [O III] 5007 emission line, for a part of the field around the Trapezium stars. The strong horizontal and vertical stripes show the influence of the per-slice sampling

\footnotetext{
${ }^{11}$ We consistently use reference wavelengths from the NIST database (Kramida et al. 2014) for this plot, e.g., $6562.819 \AA$ for $\mathrm{H} \alpha$ and $9229.014 \AA$ for Pa9.
} 


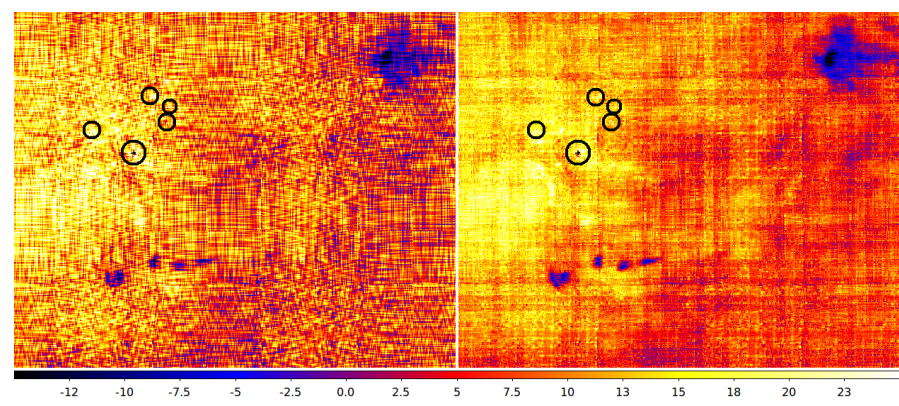

Fig. 5. Velocity field as traced by the line [O III] 5007 in part of the MUSE field of view. Left: using the LR|1.25 cube; right: using the HR $\mid 0.85$ cube. The linear color scale is $v_{\text {bary }}=-15 \ldots 25 \mathrm{~km} \mathrm{~s}^{-1}$, the brightest stars of the Trapezium cluster are marked as black circles.

in the MUSE field of view on the derived velocity field. This effect is much reduced in the HR $\mid 0.85$ cube, where the higher sampling of the line profile allows for more stable line fits. Only a residual pattern of the IFU structure is still visible (about 12 pixel wide stripes). For derivation of spatial velocity fields, it is therefore recommended to use the HR $\mid 0.85$ cube.

To determine if the blue wavelength calibration problem discussed above is an absolute offset with wavelength or has gradients across the field, we also compute average and standard deviation of velocity difference maps for a few lines: we find $v(\mathrm{H} \alpha)=$ $14.5 \pm 5.9 \mathrm{~km} \mathrm{~s}^{-1}$ (LR|1.25) and $14.4 \pm 4.5 \mathrm{~km} \mathrm{~s}^{-1}$ (HR|0.85), so that the overall velocity field in $\mathrm{H} \alpha$ is closely comparable between the two datacubes. Taking the statistics from the difference map of $\mathrm{H} \alpha$ and $\mathrm{Pa} \zeta$, we find $v(\mathrm{H} \alpha)-v(\mathrm{~Pa} \zeta)=-1.1 \pm 9.6 \mathrm{~km} \mathrm{~s}^{-1}$ $(\mathrm{LR} \mid 1.25)$ and $-1.0 \pm 8.3 \mathrm{~km} \mathrm{~s}^{-1}$ (HR|0.85), i.e., a very good agreement between velocities derived from $\mathrm{H} \alpha$ and the Paschen lines across the whole field of view. Comparing $\mathrm{H} \alpha$ and $\mathrm{H} \beta$ in the same way, we find a similar offset to the value discussed above for one slit position: $v(\mathrm{H} \alpha)-v(\mathrm{H} \beta)=4.9 \pm 10.5 \mathrm{~km} \mathrm{~s}^{-1}$ (LR 1.25$)$ and $4.9 \pm 9.4 \mathrm{~km} \mathrm{~s}^{-1}$ (HR|0.85). We therefore conclude that the relative wavelength calibration across the field is very stable when measuring each emission line individually.

We also briefly compare the map shown in Fig. 5 with the literature. The velocities we measure are at odds with those measured by Rosado et al. (2001) using an optical Fabry-Pérot instrument, who find strongly blueshifted velocites (up to and around $-100 \mathrm{~km} \mathrm{~s}^{-1}$ ) over much of the nebula, in the $\mathrm{H} \alpha$ line. In the vicinity of $\mathrm{HH} 203$ they also find velocities of around $-75 \mathrm{~km} \mathrm{~s}^{-1}$ in the $\mathrm{H} \alpha$ line, whereas we see $v \approx-20 \mathrm{~km} \mathrm{~s}^{-1}$ at the most blueshifted part of the same jet (also see Fig. 28). Our measurements are more closely comparable to the velocities measured in the near-infrared by Takami et al. (2002, with a different instrument also called MUSE), who see approximately $0 \mathrm{~km} \mathrm{~s}^{-1}$ in the region around the Trapezium stars, and $\sim-20 \mathrm{~km} \mathrm{~s}^{-1}$ in HH 203 in He I 10830 and approximately $-15 \mathrm{~km} \mathrm{~s}^{-1}$ in $\mathrm{Pa} \beta$.

Since our data are of lower spectral resolution than some other studies, we should be sensitive only to high-velocity features in the primary component of each emission line. Since strong bipolarities as reported by e.g., Doi et al. (2004) around some proplyds are only detectable in fainter components - which in our data are blended with the main line profile - we cannot detect such features.

\subsection{Absolute fluxes}

There are only a few publications on the Orion Nebula which also quote absolute emission line fluxes; most rely on flux ratios. To check the flux calibration of our data, we extracted spectra

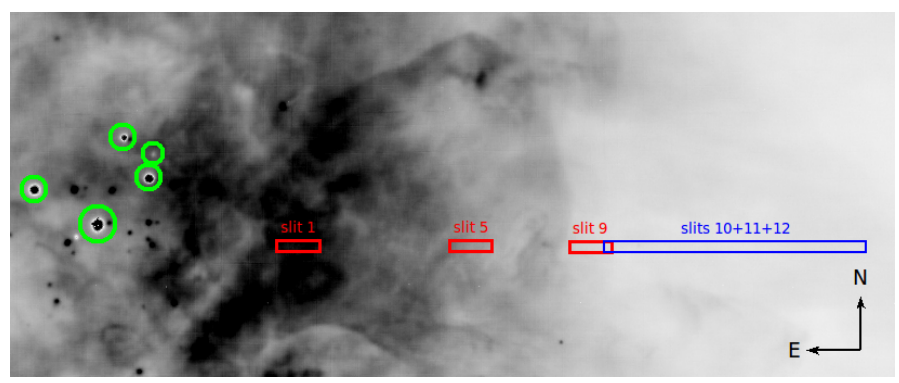

Fig. 6. Literature slit positions taken from Baldwin et al. (1991) superposed on a $\mathrm{H} \beta$ flux map. The compass has an extent of $10^{\prime \prime}$, the Trapezium stars are marked with green circles.

Table 3. Comparison of $\mathrm{H} \beta$ flux measurements with the literature.

\begin{tabular}{lccc}
\hline \hline Slit & $F_{\mathrm{H} \beta \text {,literature }}$ & $F_{\mathrm{H} \beta, \text { MUSE }}$ & Rel. change \\
\hline B91 $^{a}$ slit 10+11+12 & $1.73 \times 10^{-13}$ & $1.623 \times 10^{-13}$ & $6.2 \%$ \\
c.f. ODH10 $^{b}$ & $1.90 \times 10^{-13}$ & $1.623 \times 10^{-13}$ & $14.6 \%$ \\
\hline B91 slit 1 & $9.99 \times 10^{-13}$ & $1.229 \times 10^{-12}$ & $-23.0 \%$ \\
B91 slit 5 & $7.39 \times 10^{-13}$ & $5.314 \times 10^{-13}$ & $28.1 \%$ \\
B91 slit 9 & $2.22 \times 10^{-13}$ & $2.210 \times 10^{-13}$ & $0.5 \%$ \\
\hline B91 slit 9, 2" $\mathrm{E}^{c}$ & $2.22 \times 10^{-13}$ & $2.532 \times 10^{-13}$ & $-14.1 \%$ \\
B91 slit 9, 2" $\mathrm{W}^{c}$ & $2.22 \times 10^{-13}$ & $2.064 \times 10^{-13}$ & $7.0 \%$ \\
B91 slit 9, 2" $\mathrm{N}^{c}$ & $2.22 \times 10^{-13}$ & $2.136 \times 10^{-13}$ & $3.8 \%$ \\
B91 slit 9, 2" $\mathrm{S}^{c}$ & $2.22 \times 10^{-13}$ & $2.273 \times 10^{-13}$ & $-2.4 \%$ \\
\hline
\end{tabular}

Notes. ${ }^{(a)}$ B91 = Baldwin et al. $(1991) ;{ }^{(b)}$ observations by O'Dell \& Harris (2010) at position of B91; ${ }^{(c)}$ value measured by B91 in their slit 5 as reference.

at positions given in the literature. For this purpose, we used the REGIONS functionality of DS9, which allowed easy interactive placement of rectangular boxes onto the WCS positions, approximating the given slit lengths and widths. Then, the $3 \mathrm{D}$ plot function was used to create spectra summed over these regions.

Following the example of O'Dell \& Harris (2010, hereafter ODH10), we first extracted a 96 .' $^{\circ}$ box corresponding to the corrected position ( $4^{\prime \prime}$ south of $\theta^{1}$ Ori C as described in ODH10) for slits 10+11+12 of Baldwin et al. (1991, hereafter B91). The positions of the slits reproduced on top of the MUSE data are displayed in Fig. 6. We then measured the principal emission lines for comparison using Gaussian fits with SPLOT in IRAF ${ }^{12}$ We find $F_{\mathrm{H} \beta}=1.623 \times 10^{-13} \mathrm{erg} \mathrm{s}^{-1} \mathrm{~cm}^{-2} \operatorname{arcsec}^{-2}$, i.e., a $6 \%$ difference to B91 $\left(1.73 \times 10^{-13}\right.$ but a $14.6 \%$ difference to the measurement of $1.90 \times 10^{-13}$ by ODH10).

We carried out the same comparison for slits 1,5 , and 9 of B91, similarly correcting the position in declination. The results can be found in Table 3. The differences in absolute flux measurement are up to $28 \%$, unexpectedly high, if the positions were recovered perfectly. However, the discussion in ODH10 shows that the positions and widths used during long-slit spectroscopic observations are rather uncertain, errors of up to a few arcseconds may occur. We therefore briefly investigate what the effect of small shifts applied to the best recovered position is on the absolute fluxes of the $\mathrm{H} \beta$ line. Applying $2^{\prime \prime}$ (the width of the B91 slit) offsets to the position of slit 9 (the one that best recovers the flux determined by B91) in all four directions shows that flux differences of up to $\sim 15 \%$ can easily occur.

12 A Voigt profile gives better fits to the wings of the lines, but the integrated fluxes are typically only $\lesssim 1 \%$ larger. For simplicity and consistency with other parts of this paper, we therefore use Gaussian profiles. 
Table 4. Comparison of emission line ratios to the literature.

\begin{tabular}{|c|c|c|c|c|}
\hline $\begin{array}{l}\lambda \text { ID } \\
{[\AA]}\end{array}$ & B $91^{a}$ & $\begin{array}{l}F / F_{\mathrm{H} \beta} \\
\mathrm{ODH} 10^{b}\end{array}$ & MUSE $^{c}$ & $\begin{array}{l}\text { Rel.change } \\
\frac{\text { B91 }}{\text { MUSE }} \frac{\text { ODH10 }}{\text { MUSE }}\end{array}$ \\
\hline $4861.48 \mathrm{H} \beta$ & 1.00 & 1.00 & 1.000 & $0.0 \% \quad 0.0 \%$ \\
\hline 4959.09 [O III] & 0.92 & 0.93 & 0.934 & $-1.5 \%-0.4 \%$ \\
\hline 5007.03 [O III] & 2.76 & 2.75 & 2.813 & $-1.9 \%-2.3 \%$ \\
\hline $6563.08 \mathrm{H} \alpha$ & 3.34 & 3.20 & 3.358 & $-0.5 \%-4.9 \%$ \\
\hline $6716.81[\mathrm{~S} \mathrm{II}]$ & 0.061 & 0.058 & 0.057 & $5.9 \% \quad 1.0 \%$ \\
\hline 6731.23 [S II] & 0.076 & 0.068 & 0.071 & $6.5 \%-4.5 \%$ \\
\hline $7065.56 \mathrm{He} \mathrm{I}$ & 0.047 & 0.048 & 0.049 & $-4.8 \%-2.6 \%$ \\
\hline
\end{tabular}

Notes. ${ }^{(a)}$ Flux ratio from Baldwin et al. (1991) as quoted by ODH10; (b) flux ratio from O'Dell \& Harris (2010, their Table 1); ${ }^{(c)}$ flux measured in MUSE data in the same area using Gaussian line fits.

We therefore conclude that the main cause of the differences in absolute $\mathrm{H} \beta$ flux with respect to literature values are likely caused by uncertainties in the slit positions we can recover.

\subsection{Flux ratios}

Flux ratios, relative to $\mathrm{H} \beta$ or $\mathrm{He}$ I 6678 are given in many publications on the Orion Nebula. However, it is not always straightforward to reproduce the slit placement accurately enough to derive a meaningful comparison. For example, the observations carried out by Osterbrock et al. (1992) and Baldwin et al. (2000) were located in regions of strong emission-line gradients. Additional problems, like the unknown effect of atmospheric refraction on the literature line ratios make the comparison even more difficult.

Finally, we reproduced the approach and slit placement of O'Dell \& Harris (2010, ODH10), which has the advantage of being located in an area with shallower gradients. This also lets us compare again the relevant lines with Baldwin et al. (1991, B91).

We used our extracted spectrum and the measurement procedure that we already discussed in Sect. 3.5. The result is presented in Table 4 as fluxes relative to the $\mathrm{H} \beta$ line. The MUSE result lies approximately between the measurements of B91 and ODH10, with maximum deviations of up to $6.5 \%$ for one of the references, with a maximum deviation of $3.7 \%$ to the mean of both reference measurements. This is the deviation expected given the flux calibration accuracy quoted in Sect. 2.

Since the night was photometric, we made no attempts to check other regions of our cube, but assume that the relative fluxes are accurate to $\sim 5 \%$ over the full field.

\section{Artifacts visible in the data}

Potential users of the data should be aware of a few artifacts present in the data. Some of them are due to the imperfect calibrations taken at the time of the first commissioning with the MUSE instrument, others are instrumental features that are hard to model and hence cannot be removed. These effects will be described in detail by Bacon et al. (in prep.).

The brightest part of the data, the region around the Trapezium is particularly affected. Figure 7 shows this region as a color composite, highlighting the continuum in three wavelength bands.

At all wavelengths, ghosts around bright objects are visible as vertical and horizontal stripes. These are likely due to internal reflections in the spectrographs, resulting in a faint background

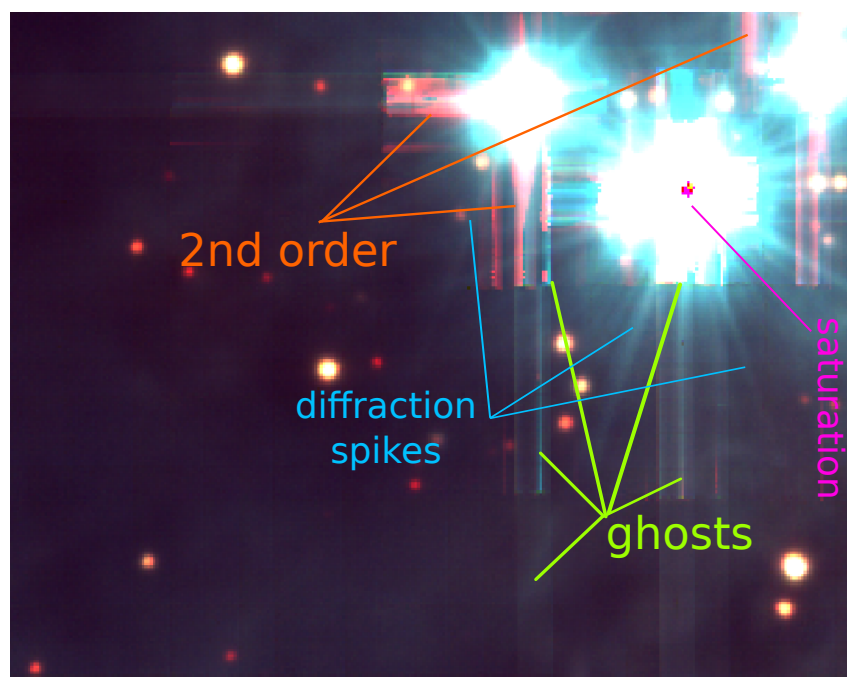

Fig. 7. Color composite of the region near the Trapezium stars. The colors are created from continuum regions of the spectrum, with blue: $4600 \ldots 4800 \AA$ A, green: $5300 \ldots 5500 \AA$, and red: $9100 \ldots 9200 \AA$. Ghosts are visible as blueish vertical and horizontal stripes, second order as reddish striping, and saturation as magenta pixels.

(at the level of $6 \times 10^{-6}$, Bacon et al., in prep.) across the CCD, mostly affecting the slice in which the bright object is located but also neighboring slices. The cross-pattern this creates is due to the observations with the two position angles of 0 and $90 \mathrm{deg}$. We note that these ghosts are not the same as the usual diffraction spikes seen in pure imaging data. These spikes exist in the MUSE data as well (see Fig. 7), but are fainter than the ghosts, and smoothly decrease with radial distance from the star.

In the very red part of the spectrum, the second spectral order also becomes apparent. Since this order is unfocused in MUSE, it appears broadened compared to real features in the data. In Fig. 7 the second-order can be seen spatially, as red striping, again in horizontal and vertical directions. The second order is also visible in spectral direction, as displayed in Fig. $8^{13}$. The nebular continuum is almost featureless, so this merely creates an additional offset, but strong emission lines in the blue can create broad bumps in the spectrum. However, if care is taken to locally fit or subtract the background when integrating line fluxes, these broad bumps should not cause any problems when extracting signals from the cube.

The brightest stars are also saturated near their peak. Part of this saturation appears as magenta pixels in Fig. 7. However, around the strongly saturated pixels, a few more pixels may be strongly negative, or have positive values below the real value. No attempts were made to mask these out.

Since neither telluric emission (Fig. 8) nor absorption (Fig. 14) were treated in our reduction, both still show up in extracted spectra. The nebular emission towards the Huygens region is very bright compared to the sky, most measurements are unaffected by more than a few percentage points. Notable exceptions are emission lines that are of comparable brightness to the sky background, such as [O I] 5577 or - in a few places where the nebula is faint - [OI] 6300 .

When extracting spectra of (bright) point sources from the HR|0.85 MUSE cube, wiggles in the continuum can be seen

\footnotetext{
13 The second order is not correctly wavelength calibrated, nor does it appear exactly on the same horizontal (or spatial) position on the CCD. This likely causes the bumps to appear somewhat offset from the expected position of $2 \times \lambda_{1}$.
} 


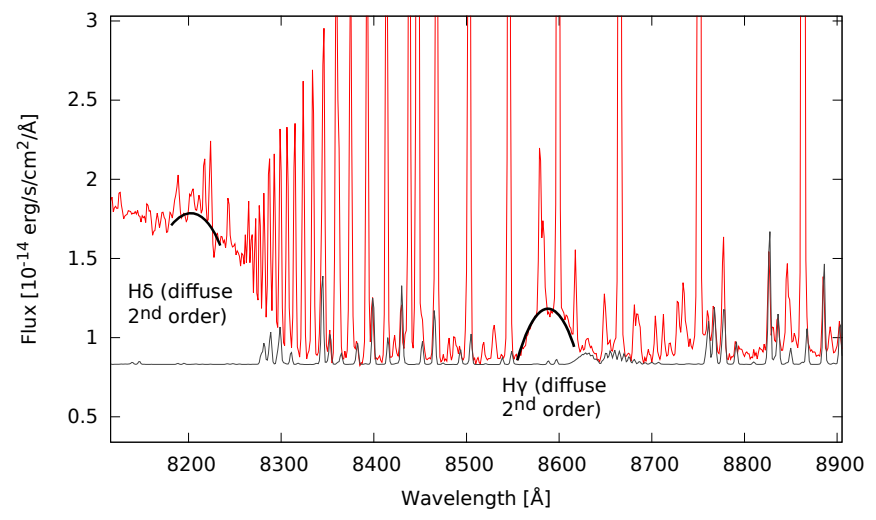

Fig. 8. Spectral region around the Paschen limit. The red spectrum was extracted from the MUSE cube at the approximate position of slit 1 of Baldwin et al. (1991). In gray, a typical Paranal sky spectrum is shown. Broad second-order contamination by prominent blue Balmer emission lines is marked in black.

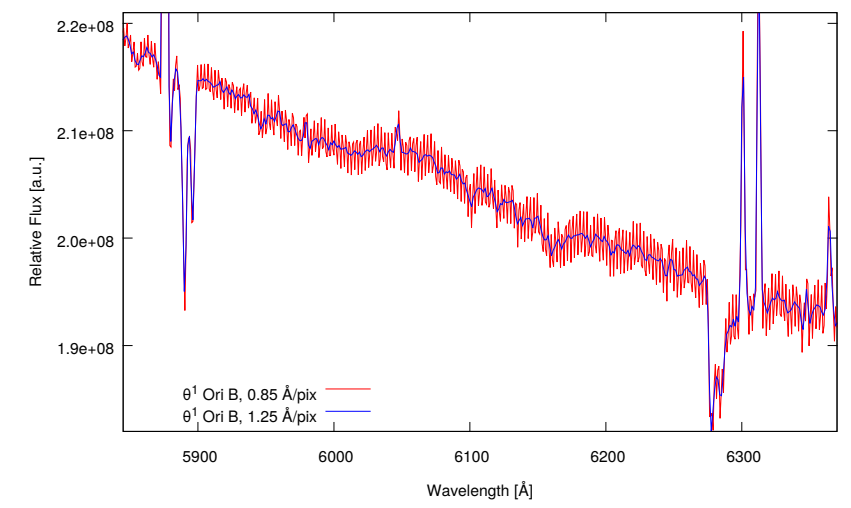

Fig. 9. Wiggles in the extracted HR|0.85 spectrum of $\theta^{1}$ Ori B compared with normal LR|1.25 spectrum.

(Fig. 9). The origin of this feature is not fully understood, but it is likely caused by a change in the spatial point-spread function (PSF), i.e., variations in seeing between exposures. Since the two exposures per position are interleaved in the final HR $\mid 0.85$ cube and - depending on the exact sampling in the cube - often contribute to alternating wavelength planes, any variations in seeing between exposures also effectively causes the PSF to vary between adjacent planes in the cube. When integrating the flux of a star in an aperture that is too small to integrate the whole flux in both seeing conditions, the output spectrum looks particularly wiggly, as displayed in Fig. 9. The problem is mitigated when using PSF-matched spectral extraction (Kamann et al. 2013) where the PSF is fitted to each wavelength plane separately instead of using simple aperture extraction. We confirmed this by testing extraction strategies in a different MUSE two-exposure dataset (of a globular cluster, Kamann et al., in prep.) at LR $\mid 1.25$ and HR $\mid 0.85$ output sampling. However, since the PSF fit is imperfect, even then the wiggles do not completely disappear from the HR $\mid 0.85$ data. For stellar work, we therefore recommend use of the LR|1.25 cube where this problem does not occur at all. We were unable to detect this problem in the ionized gas. Since the spatial changes in the gaseous nebula are smoother, this problem only affects point sources but not the gas continuum or the emission lines.

\section{Analysis of the ionized gas}

This section is devoted to showcase analysis methods that are possible with the MUSE data. All maps we show here were created using Gaussian fits to single emission lines. The fit was carried out on the LR|1.25 data, separately in each spaxel (using the HR $\mid 0.85$ data gives almost identical results). We allowed variations in the flux of the line, its width, and the central position (restricted to be within $5 \AA$ of the expected zero-redshift center). The fit also included a constant background offset. Since we did not attempt to mask stars before the fit, these show up as artifacts in some of the maps, especially for Balmer lines.

At the spectral resolution of MUSE, the [O I] 6300 line cannot be separated from the sky line at the same wavelength. We therefore assumed that the nebular line dominates the emission, but in the region of the Dark Bay (where the nebular line is weak) and especially in MUSE exposures 31 to 36, the sky line was stronger, leading to increased flux in the area covered by these exposures. We modeled this as a linear multiplicative gradient, decreasing from 1.34 at the left edge of the field to 1.0 at the approximate right edge of exposure $31+32$ (cf. Fig. 1), and divided it out. Since we ignored other lines that coincide with telluric features, [O I] 6300 is the only line that was treated in this special way.

In Figs. 10 to 13 we show different color representations, using the fluxes of three emission lines at a time extracted from the MUSE data. In Fig. 10, the selected lines trace different ionization states in the main ionization front and hence different distances from $\theta^{1}$ Ori C (O'Dell 2001): [S II] emission is produced in the layer of the main ionization front on top of the molecular cloud, [N II] at intermediate distances, and $\mathrm{H} \beta$ directly around the Trapezium cluster. The most striking features are the Bright Bar that runs across the image at the bottom left, and the Orion-S region - the brightest part of the nebula close to the main ionizing source - in the center. Since the stars are visible only as small and faint artifacts in this image, in the region to the SE of the Bright Bar, only a few Herbig-Haro objects are prominently visible: the jets of HH 203 and HH 204 (O'Dell \& Wen 1994) next to each other and the more roundish blob of HH 524 (Bally et al. 2000). In this image, we also note a spot with strongly enhanced [S II] emission in the upper right that appears as a red clump in Fig. 10. This is HH 201, one of the "bullets" from the wide-angle Orion outflow (Graham et al. 2003; Bally et al. 2015). Compared to the surrounding material, it is similarly bright in [O I] 6300. In the MUSE data, we detect a secondary (blueshifted) component in the velocity field of these emission lines in this region.

Figure 11 shows the same image but corrected for extinction using the Balmer decrement (see Sect. 5.1). Fig. 12 combines the emission line fluxes of three hydrogen lines spread over almost the full MUSE wavelength range: Paschen9 (=Pa $\zeta)$ at $9229.7 \AA$, $\mathrm{H} \alpha 6562.8 \AA$, and $\mathrm{H} \beta 4861.3 \AA$. This is the image before correcting for extinction, the reddening-corrected version (not shown) is devoid of color.

Figure 13 shows three oxygen ions accessible using the MUSE data: [O III] 5007 represents the highest ionization state, the hottest region of the nebula, and is colored red, [O II] 7320 appears for the intermediate ionization state, and [O I] 6300 for the coldest gas. For this image we used the extinction-corrected fluxes measured for the emission lines. The different extent of the three states and the diversity in structure visible are both due to the stratification of the nebula. This is especially visible at the Bright Bar. 


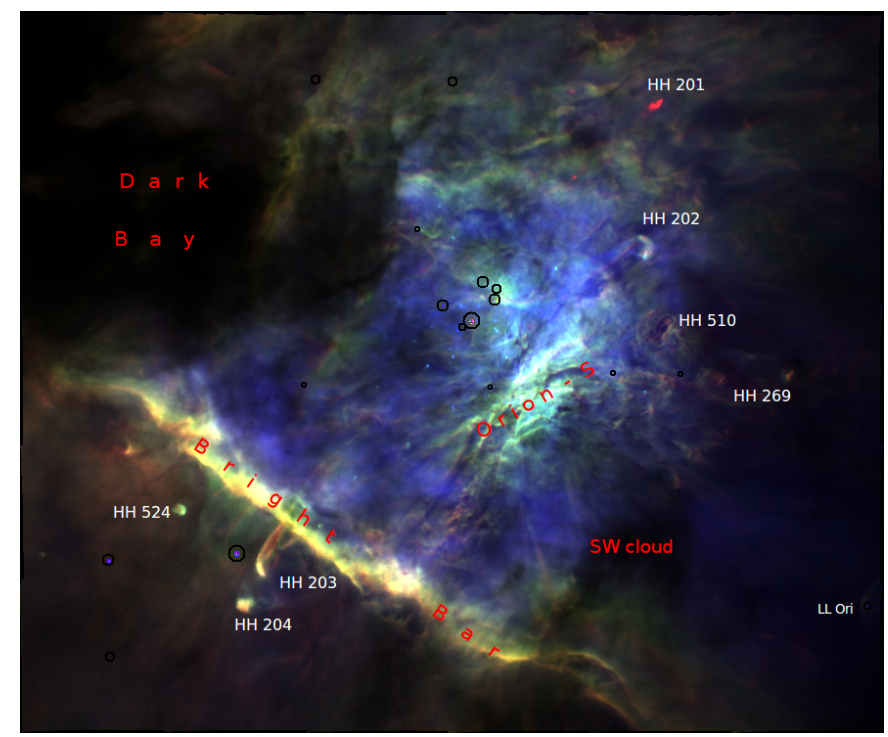

Fig. 10. Color composite using fluxes of three emission lines, with blue: $\mathrm{H} \beta$, green: [N II] 6584, and red: [S II] 6731. The brightest stars in the field are marked with black circles, large features are annotated in red text, other prominent features in white.

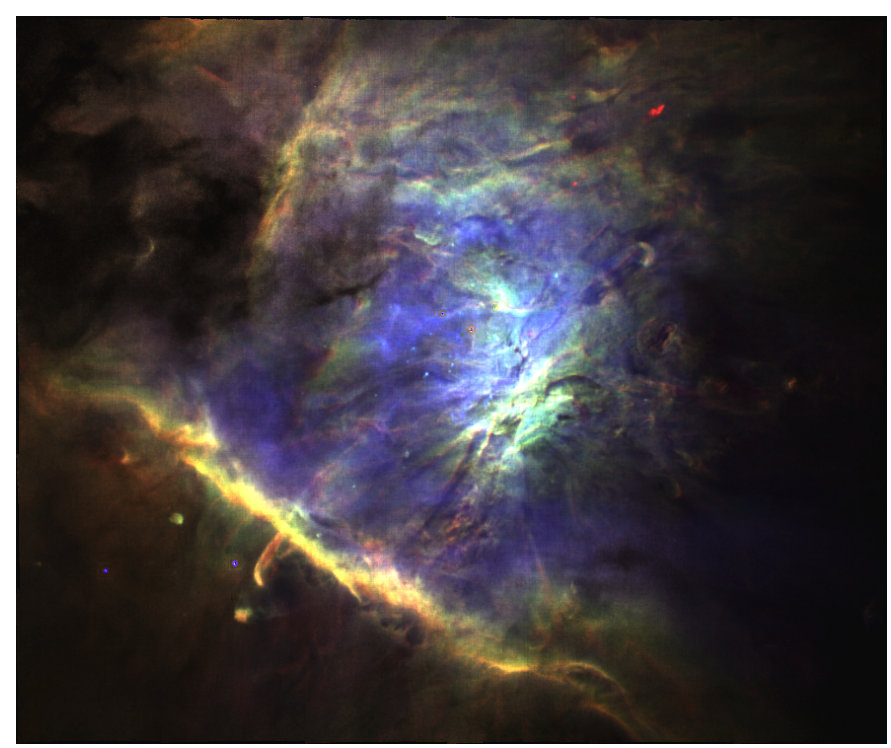

Fig. 11. Color composite, created from the same emission lines as in Fig. 10, but using the reddening-corrected fluxes an using the extinction estimate from the Balmer decrement.

Figure 14 presents two nebular spectra over the full wavelength range extracted from the MUSE data. At the blue end of the spectrum, the first visible emission line is O II 4642, but in the cube several other even fainter lines are detected. Hydrogen Paschen lines can be discriminated from $\mathrm{Pa} 9$ to $\mathrm{Pa} 35$ at the red end.

Like the extraction regions from the cube, the line identifications in Fig. 14 were taken from Baldwin et al. (1991) and Osterbrock et al. (1992). We can identify lines down to a flux of about $0.005 \times F_{\text {He I6678 }}$ listed in their reference tables. However, a few lines visible in our spectrum are not listed in these reference

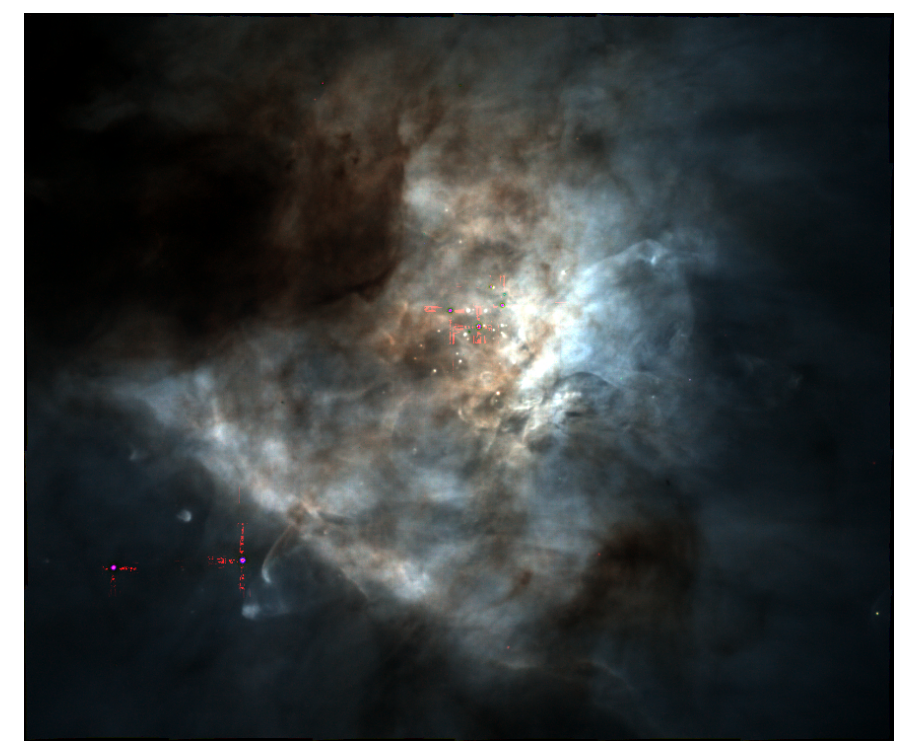

Fig. 12. Color composite, using emission line fluxes of red: Paschen 9, green: $\mathrm{H} \alpha$, blue: $\mathrm{H} \beta$. In the red band, the effect of ghosts around bright stars is visible (cf. Fig. 7).

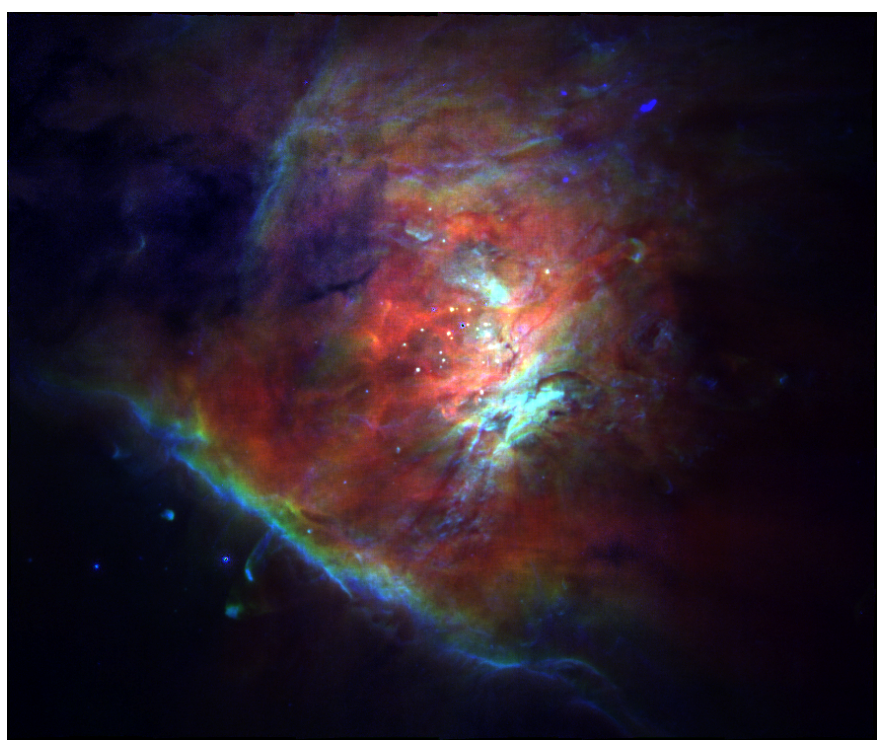

Fig. 13. Enhanced color image, using the three ionization levels of oxygen detectable with the MUSE data: red: [O III] 5007, green: [O II] 7320, blue: [OI] 6300, all corrected for extinction using the Balmer decrement. [OI] is partially corrected for sky-line contribution. This representation shows the stratification of the nebula even more clearly than Fig. 10.

sources and are not known sky emission lines. They remain unidentified and are specially marked in our figure.

As a first demonstration of what is possible with this new dataset, we analyze the nebular emission in a spatially resolved (pixel by pixel) manner.

\subsection{Extinction}

We follow O'Dell \& Harris (2010) by selecting an intrinsic $\mathrm{H} \alpha$ to $\mathrm{H} \beta$ flux ratio of 2.89 for case $\mathrm{B}$ and $T_{\mathrm{e}}=9000 \mathrm{~K}$ (see Storey \& Hummer 1995; Osterbrock \& Ferland 2005). We 

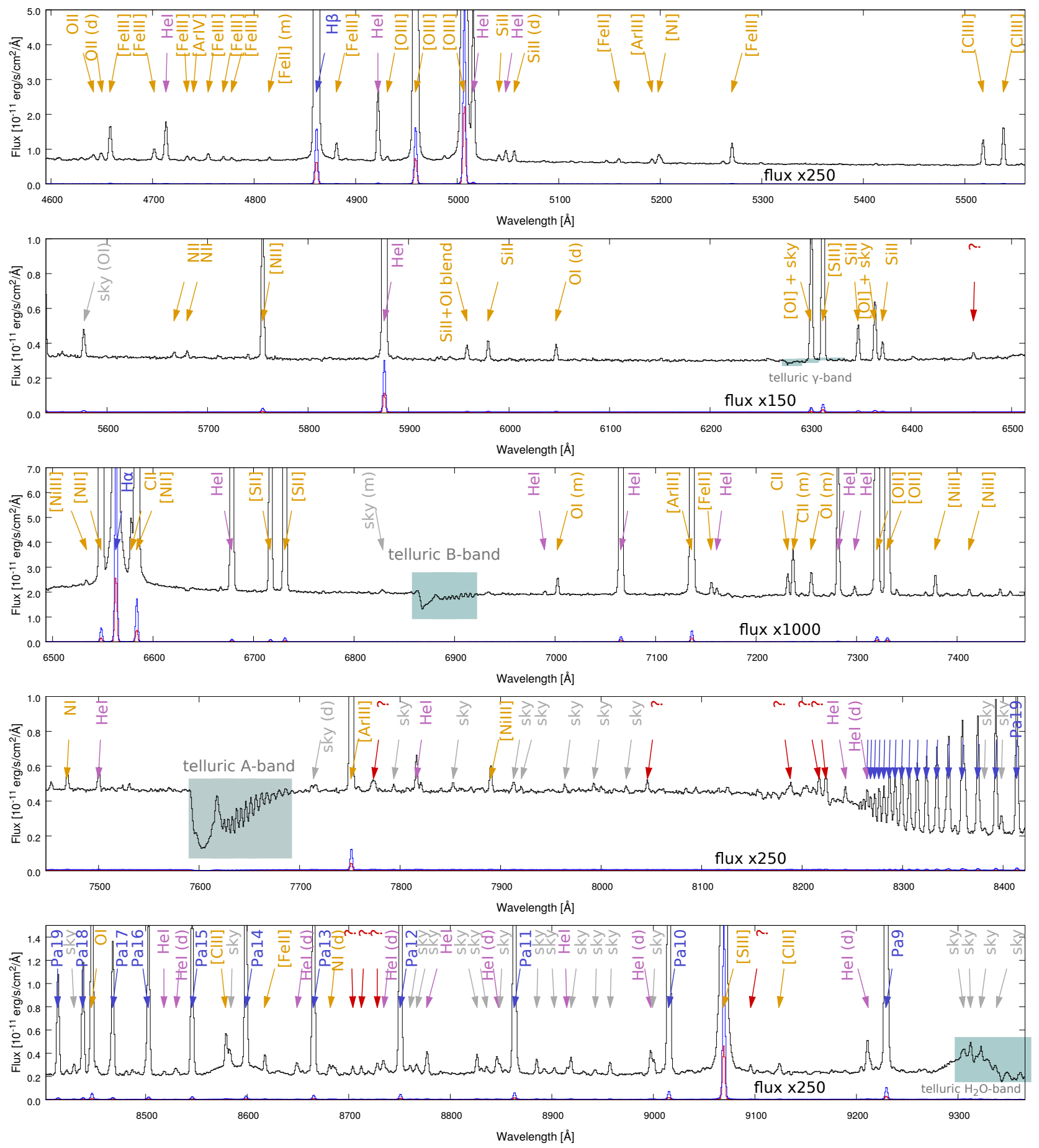

Fig. 14. Full spectrum of the MUSE dataset, extracted at the approximate positions of slit1 from Baldwin et al. (1991; red) and at the optical slit from Osterbrock et al. (1992; blue). The "slit1"-spectrum is shown again in black, multiplied with the factor at the bottom of each panel to highlight fainter lines. We note that the different panels have different absolute scales. Line identifications from the same two references are shown, hydrogen lines are in blue (lines Pa20 to Pa35 are marked but not annotated), helium lines in violet, metal lines in orange, and lines where the sky contribution dominates are gray. Unresolved line doublets and multiplets are marked with "(d)" and "(m)", respectively. A few faint lines without identification are marked in red.

interpolate the flux measurement of the Balmer lines at the positions of bright stars (where the Balmer decrement could not be measured reliably), and then input them as well as the reference value into the PYNEB software (v1.0.9, Luridiana et al. 2013, 2015), and derive a reddening map using the extinction curve of Cardelli et al. (1989) as refined for the Orion Nebula by Blagrave et al. (2007) with $R_{V}=5.5$. This map is displayed in Fig. 15 as $c_{\mathrm{H} \beta}$. The contour levels displayed there correspond to $0.9 \lesssim A_{V} \lesssim 2.7 \mathrm{mag}$ or - when applying the relation of Bohlin et al. (1978) - to column densities of $9.6 \times 10^{20} \lesssim N\left(\mathrm{HI}+\mathrm{H}_{2}\right) \lesssim 2.9 \times 10^{21} \mathrm{~cm}^{-2}$. As expected, the regions of the Dark Bay and the SW cloud (O'Dell et al. 2009) show the strongest extinction while for example the region southeast of the Bright Bar as well as the western arcminute of our field exhibit very moderate reddening. By contrast with (sub)millimeter emission maps (e.g., Johnstone \& Bally 1999), 


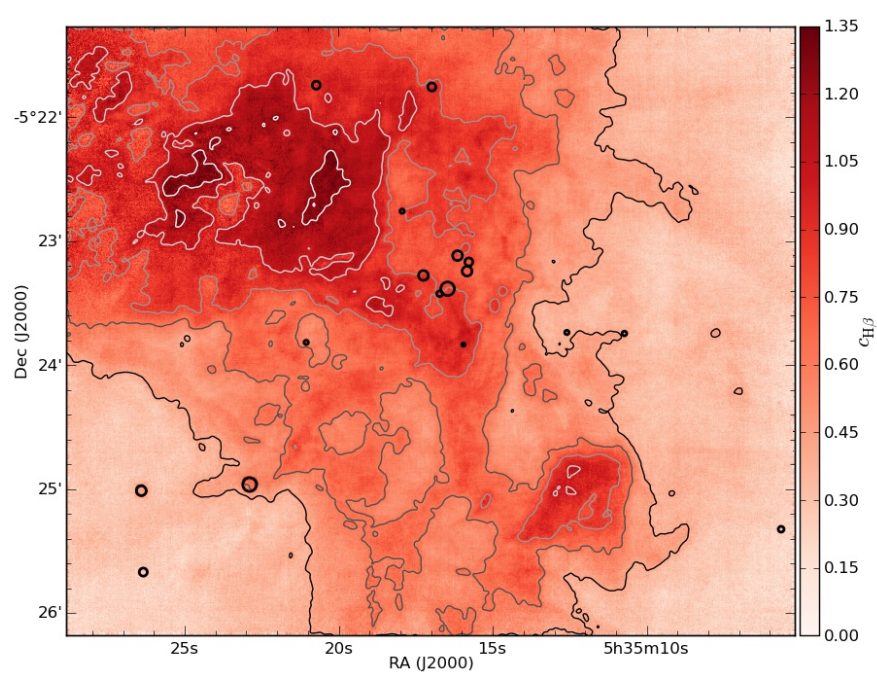

Fig. 15. Extinction map of the central Orion Nebula, as derived from the $\mathrm{H} \alpha$ to $\mathrm{H} \beta$ flux ratio, displayed as $c_{\mathrm{H} \beta}$. Bright stars are marked with black circles, the smoothed contours are $0.4 \leq c_{\mathrm{H} \beta} \leq 1.2$ in steps of 0.2 .

the foreground dust structure appears smooth and devoid of significant substructure. Such substructure is most commonly seen in self-gravitating gas clouds that form filamentary and clumpy structures on scales from the parent cloud down to individual protostars (e.g., Johnstone \& Bally 2006; Takahashi et al. 2013). The lack of this clumping in our extinction map suggests that this foreground material is not self-gravitating.

While qualitatively similar to the reddening map derived by O'Dell \& Yusef-Zadeh (2000) from a radio-to-optical surface brightness comparison we note that we only reach peak values of $c_{\mathrm{H} \beta}=1.35$ in the Dark Bay, whereas O'Dell \& Yusef-Zadeh found values of up to 2.0. The comparison to their Balmer decrement-derived map, however, shows very similar absolute trends over the smaller area covered by them, such that in the vicinity of the Trapezium $c_{\mathrm{H} \beta}$ reaches 0.8 , but is only about 0.5 between the SW cloud and the Trapezium.

We use this map to correct all measured emission line fluxes for reddening. We then also use the extinction-corrected flux maps to recreate a nominally dust-free version of Fig. 10 in Fig. 11, and also Fig. 13 (for which no uncorrected counterpart is shown). It is apparent from the latter image that the reddening correction based on the Balmer-decrement is imperfect, since the Dark Bay and the SW cloud get more transparent but do not disappear.

\subsection{Emission line maps}

Figures 16 to 19 show the Orion Nebula in dedicated emission lines as representatives of different ionization stages. The images of the Orion Nebula are more compact and diffuse in the higher ionized lines [O III] 5007 and [S III] 6312 than in the lower ionized lines [S II] 6731 and [O I] 6300.

The images in the low ionization lines such as [O I] are much more structured than in the higher ionization lines because the low ionization lines only form over a very narrow range of physical conditions in the nebula in comparison to the high ionization lines. Such effects are known, for example, from images of planetary nebulae taken in the light of different emission lines (Osterbrock \& Ferland 2005). Neutral gas is also often intrinsically more structured because the lower temperature, and therefore pressure, means that the neutral gas is more easily compressed.

\subsection{Diagnostic maps}

With the amount of emission lines present in the MUSE data and the reddening correction derived in Sect. 5.1, we can easily create flux ratio images, i.e., diagnostic maps.

The diagnostic images (Figs. 20 to 23) - based on different line intensity ratios - highlight different regions in the Orion nebula. The figures based on the $\mathrm{H} \alpha / \mathrm{He}$ I 6678 (Fig. 20) and $\mathrm{H} \beta /[\mathrm{O}$ III] 5007 (Fig. 22) line ratios highlight the innermost and most strongly ionized structures of the nebula surrounding the central Trapezium stars. These line ratios are mainly indicators of the mean level of ionization and temperature (Osterbrock $\&$ Ferland 2005). The discovery of a central [O III] shell surrounding the Trapezium stars has been reported by O'Dell et al. (2009), indicating a stationary high-ionization structure. We also see hints of this structure in our $\mathrm{H} \beta /[\mathrm{O}$ III] 5007 image (Fig. 22, cf. Fig. 3 of O'Dell et al. 2009) and may even detect this $\mathrm{H} \alpha / \mathrm{He}$ I 6678 map (Fig. 20). Furthermore, extended shell structures are clearly visible towards the north.

In the [O III] 5007/[N II] 6584 image (Fig. 23) the darker Orion-S region stands out southwest of the Trapezium. This foreground Orion-S complex hosts embedded stars that are sources of many large-scale optical outflows (O'Dell et al. 2009). The bright elongated region towards the outer southwest highlights the shocked wind region. Here the gas flows towards the lowdensity end in a so-called champagne flow (Arthur \& Hoare 2006). In addition, the bow shock connected to the T Tauri star LL Ori (see spectrum in Fig. 3) sticks out to the outermost southwest. Furthermore, towards the west a loop structure pops up. Similar structures can be recognized as well in highly processed $20 \mathrm{~cm}$ continuum images taken with the VLA (Yusef-Zadeh 1990).

The $\mathrm{H} \alpha /[\mathrm{OI}] 6300$ image (Fig. 21) shows sharp extended structures in the outer (cooler) regions of the Orion Nebula.

\subsection{Electron temperature/density}

We again use PYNEB to cross-iterate electron density $\left(N_{\mathrm{e}}\right)$ and electron temparature $\left(T_{\mathrm{e}}\right)$ of the ionized gas, using extinctioncorrected line ratios. We use the temperature sensitive line ratios [N II] 5755/6548, [N II] 5755/6584, and [S III] 6312/9069 together with the density sensitive ratios [S II] 6731/6716 and [Cl III] 5538/5518, and let the iteration start at $T_{\mathrm{e}}=10000 \mathrm{~K}$. The assumptions for using PyNeb are that the $n$-level approximation that this tool is based on (also see De Robertis et al. 1987, for a five-level precursor) can describe the ionized states of the gas involved, and that the gas along the line of sight is sufficiently homogeneous for the emission lines to represent the luninosity-weighted physical state of the ionized gas in each spatial element.

We derive maps of $T_{\mathrm{e}}$ from [N II] (averaged from 5755/6548 and 5755/6584, cross-iterated with [S II]) and [S III] 6312/9069 (cross-iterated with $[\mathrm{S} \mathrm{II}]^{14}$ ), and $N_{\mathrm{e}}$ from [S II] 6731/6716 and [Cl III] 5538/5518.

Since we did not attempt to disentangle stellar continuum and gas or mask positions of stars, many strong small-scale features in these maps may be artifacts. This can be easily verified

\footnotetext{
${ }^{14}$ The map cross-iterated against [Cl III] gives very similar temperatures, but has much lower quality due to many more pixels with non-converging iterations.
} 


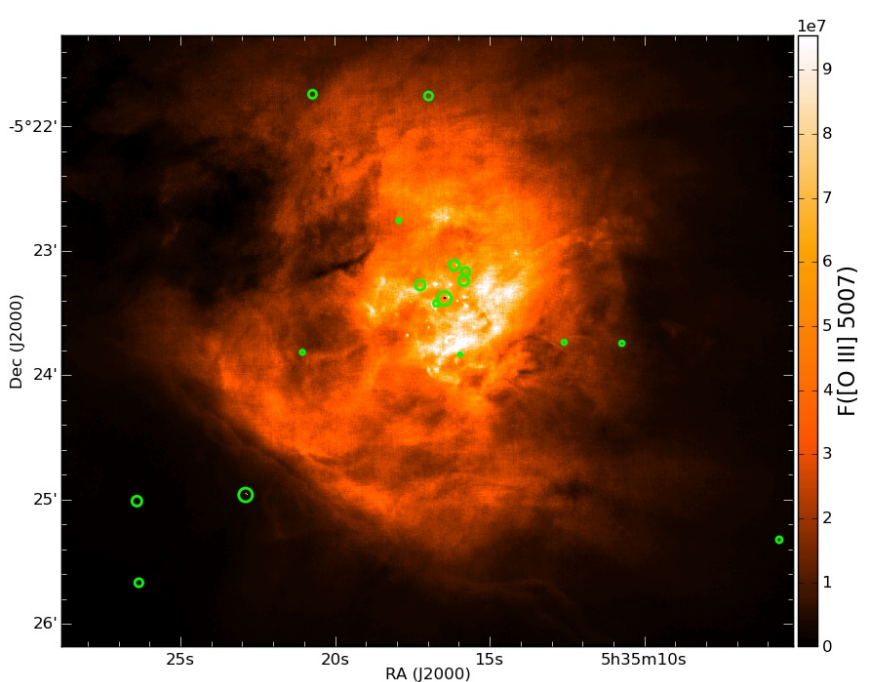

Fig. 16. Reddening-corrected flux map of [O III] 5007.

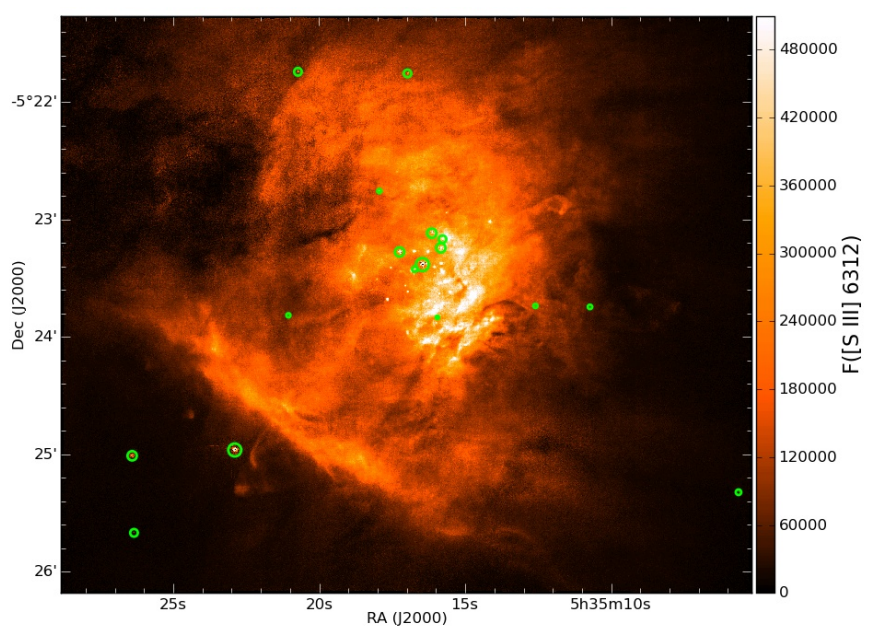

Fig. 17. Reddening-corrected flux map of [S III] 6312.

using a continuum part of the spectral range. The brightest stars are therefore marked on the corresponding maps.

In the [N II]-derived $T_{\mathrm{e}}$-map in Fig. 24 , the hottest regions are 154-309 (in the coordinate system of O'Dell \& Wen 1994), about $21^{\prime \prime} \mathrm{NW}$ from $\theta^{1}$ Ori C (marked in Fig. 24), and 140-352, $47^{\prime \prime} \mathrm{SW}$ of $\theta^{1}$ Ori C, both reaching $\sim 13000 \mathrm{~K}$. The coldest region appears to be beyond the Bright $\mathrm{Bar}$, around $\theta^{2}$ Ori $\mathrm{A}$ and $\theta^{2}$ Ori B, with $T_{\mathrm{e}} \sim 8500 \mathrm{~K}$. That $T_{\mathrm{e}}$ is found to be high in the Dark Bay may be a problem of imperfect extinction correction. A grid-like pattern is visible in this map, caused by the $T_{\mathrm{e}}$ estimate being about $200-300 \mathrm{~K}$ lower in the regions where multiple exposures overlap with adjacent pointings. This is caused by a slight systematic bias of the weaker line [N II] 5755 and can be viewed as a representation of the systematic uncertainty of these maps.

The [S III]-derived $T_{\mathrm{e}}$-map in Fig. 25 shows a different behavior from that of the $[\mathrm{N}$ II]-derived map. The hottest regions are some of the Herbig-Haro shocks, e.g., HH 204 reaching $\sim 9200 \mathrm{~K}$, and the Bright Bar and the region SW (called the "Orion-S" feature by O'Dell et al. 2009) of the Trapezium are hotter than the surrounding nebula. The coldest regions in this ionization layer with about $7800 \mathrm{~K}$ are between the Bright Bar and the Dark Bay and between the Trapezium and the Dark Bay.

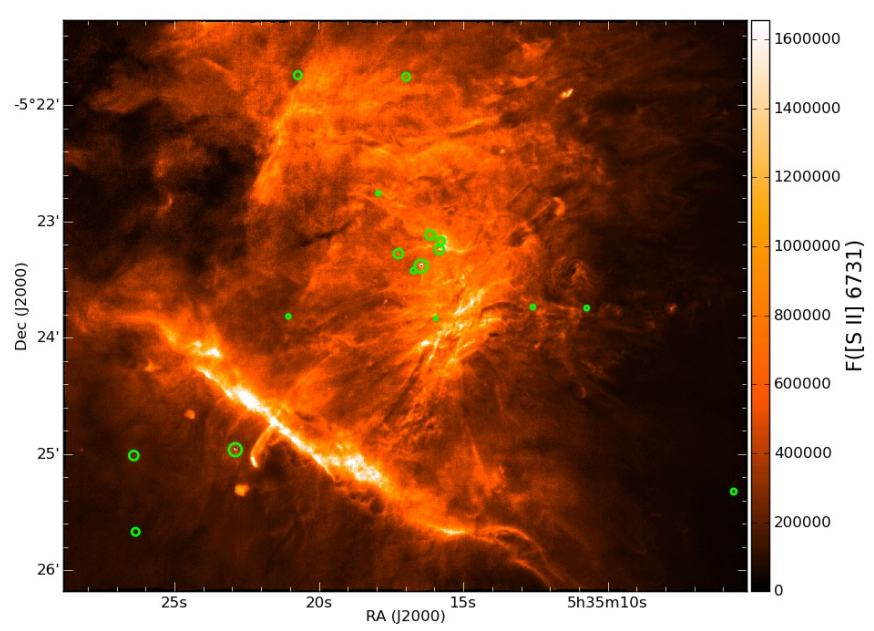

Fig. 18. Reddening-corrected flux map of [S II] 6731.

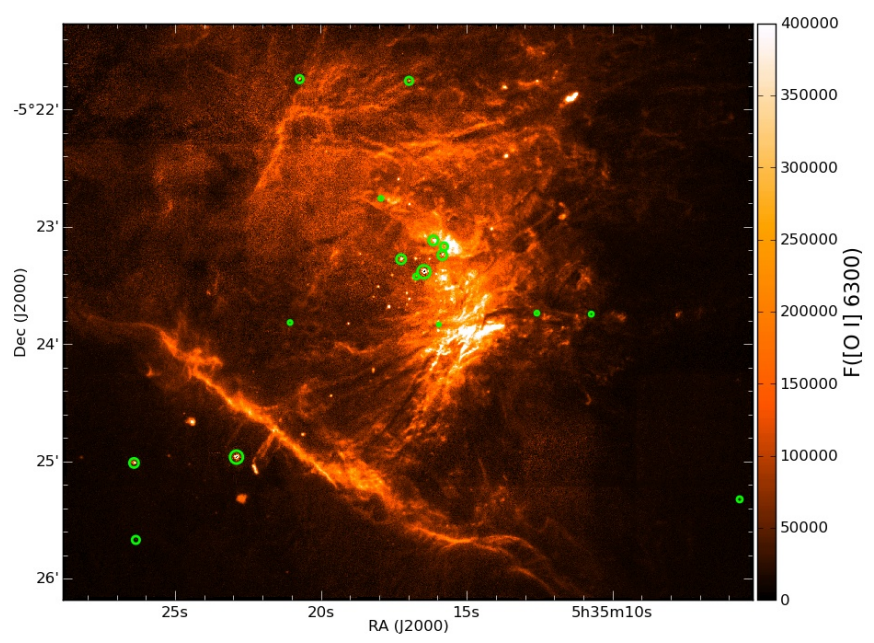

Fig. 19. Reddening-corrected flux map of [OI] 6300, partially corrected for sky-line contribution.

The [S III] lines were previously used by Foukal (1974) to derive $T_{\mathrm{e}}=9700 \pm 1000 \mathrm{~K}$ in a slit of $150^{\prime \prime} \times 15^{\prime \prime}$ in an unspecified location. Assuming that they pointed within the brightest part of the nebula but not on the Trapezium stars, their result agrees with ours. Again, the extremely high $T_{\mathrm{e}}$ values derived in the Dark Bay may be an artifact. Similar to $T_{\mathrm{e}}([\mathrm{N}$ II $])$, the gridlike structure originates in the flux measurement of the fainter line [S III] 6312.

Given the density of known sources in the Orion Nebula and changes in the absolute world coordinate systems used in the literature, it is not always clear which known objects are related to features in our data. However, at least a few of the compact high- $T_{\mathrm{e}}$ peaks visible in Figs. 24 and 25 around the location of the Trapezium cluster can be identified with known young stellar objects and proplyds, e.g., 170-337 (O'Dell \& Wen 1994), d141-301, and j177-341 (Bally et al. 2000).

The electron density as derived using the [S II] doublet (see Fig. 26) is consistent with the map derived by Pogge et al. (1992), but shows features with higher spatial resolution. The value of $N_{\mathrm{e}}$ varies between $\sim 500 \mathrm{~cm}^{-3}$ at the edge of the field and densities in excess of $10000 \mathrm{~cm}^{-3}$ in the Orion-S region.

The layer of the ionization front showing [Cl III] emission has an even higher density, reaching up to $N_{\mathrm{e}} \approx 25000 \mathrm{~cm}^{-3}$ 


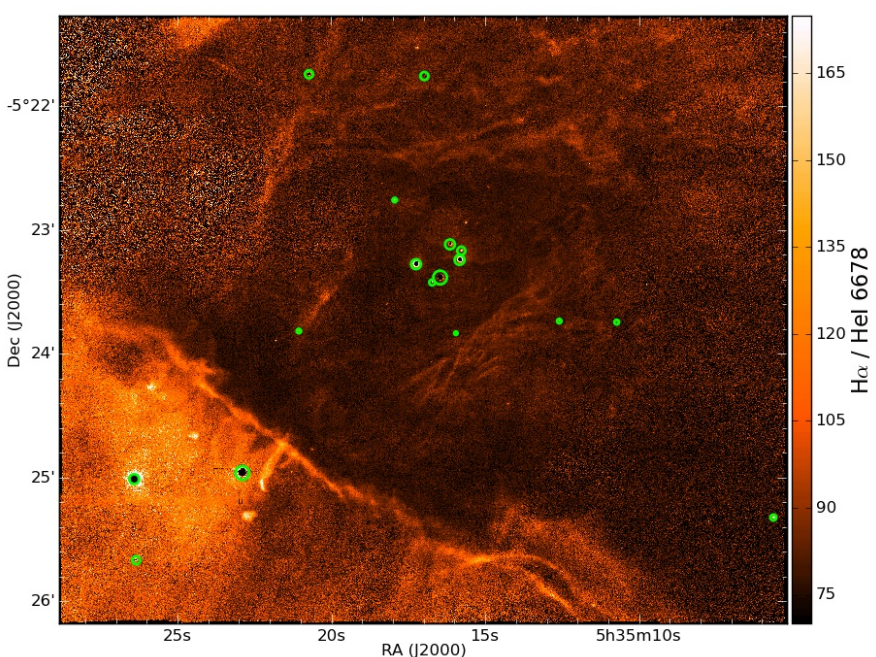

Fig. 20. Reddening-corrected emission line ratio map of $\mathrm{H} \alpha / \mathrm{He}$ I 6678.

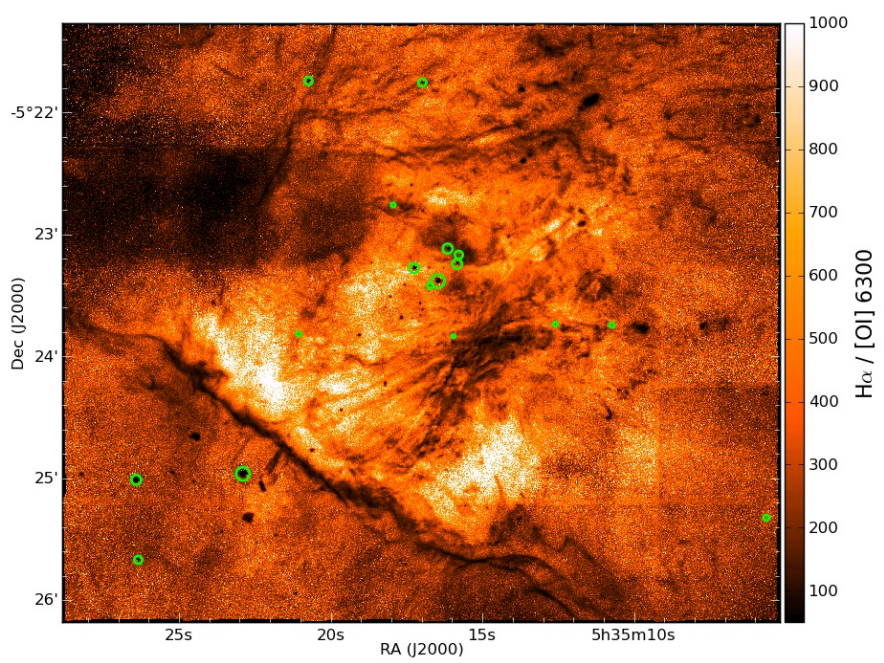

Fig. 21. Reddening-corrected emission line ratio map of $\mathrm{H} \alpha /[\mathrm{O} \mathrm{I}] 6300$, partially corrected for sky-line contribution to [O I] 6300.

in parts of the Orion-S region, as shown on Fig. 27. The lowest values derived from the [Cl III] doublet give $N_{\mathrm{e}} \approx 4000 \mathrm{~cm}^{-3}$, just north of the Bright Bar. That $N_{\mathrm{e}}$ estimated from [Cl III] gives higher densities than when derived from [S II] was qualitatively already presented by Núñez-Díaz et al. (2013). However, their scale does not allow a direct comparison to our map. For most of the field of view, the densities from [ $\mathrm{Cl} \mathrm{III}]$ are too noisy to distinguish more than genereral trends with position.

The range in derived electron temperatures and densities prompted Sánchez et al. (2007) to compute the extinction values at each position using the physical gas conditions inferred by them. However, the dependency of the Case B Balmer decrement on the densities is weak (for the values derived here, it only changes by $1 \%$ ). Since the temperature estimate is not independent of the reddening correction, we would need to add another layer of iterations. The expected changes are small (changes in the derived Balmer decrement reference value are at most $3 \%$ ), we therefore prefer to not carry the analysis beyond this point.

\subsection{Velocity field}

Figure 28 shows the velocity field recovered from Gaussian centroids of the emission lines $\mathrm{H} \alpha$ (absolute velocities against

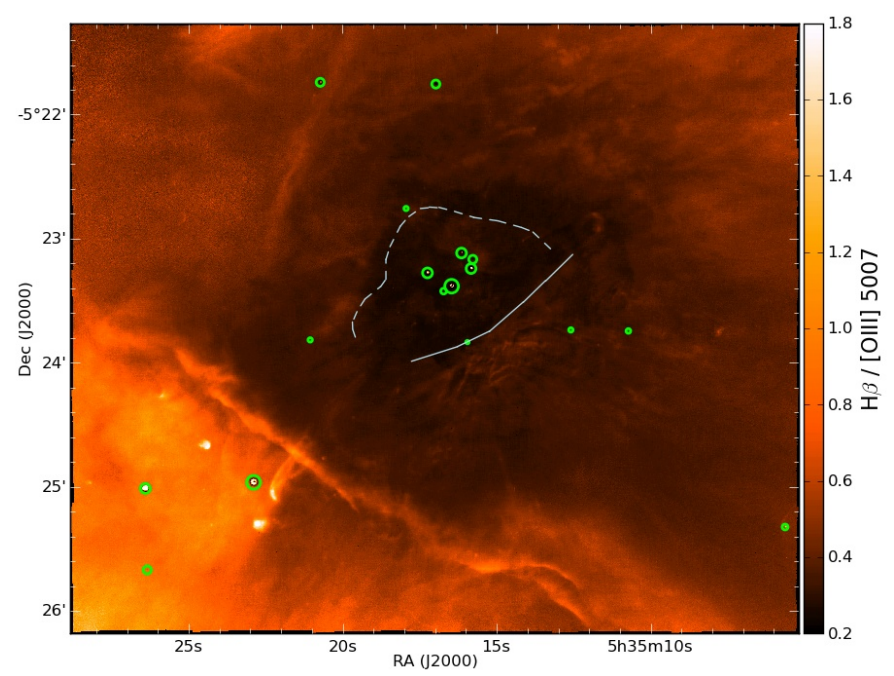

Fig. 22. Reddening-corrected emission line ratio map of $\mathrm{H} \beta /[\mathrm{O}$ III $]$ 5007. The [O III] shell and the SE-NW ionization boundary discussed by O'Dell et al. (2009) are marked.

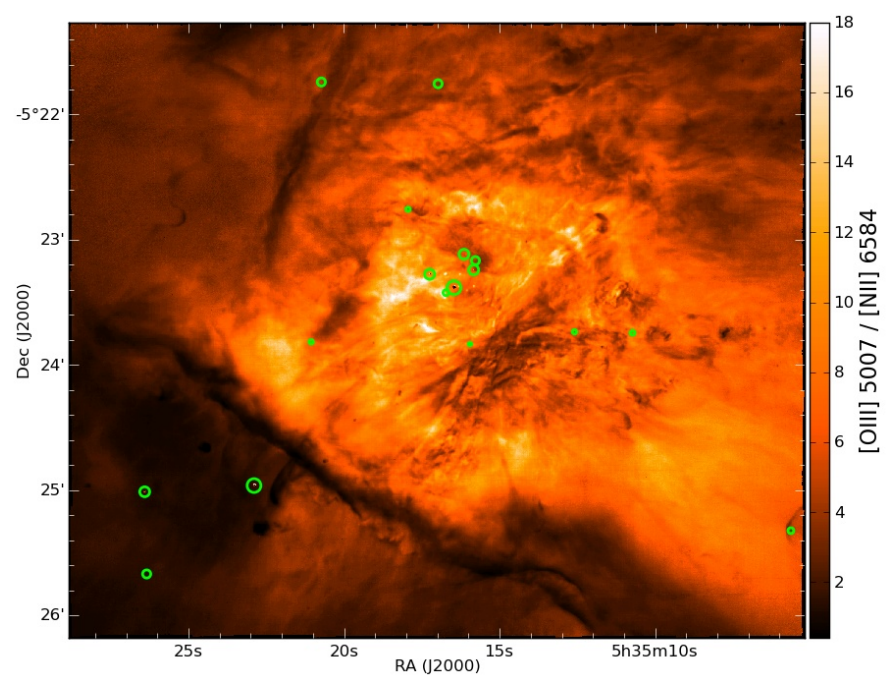

Fig. 23. Reddening-corrected emission line ratio map of [O III] 5007/[N II] 6584 .

barycentric zeropoint), [N II] 6584 (relative to $\mathrm{H} \alpha$ ), and [S II] 6731 (relative to [N II]) for the full field mapped by MUSE.

The strongest features in velocity space for $\mathrm{H} \alpha$ are the Herbig-Haro jets, especially the blueshifted objects HH202, HH 269, HH 203, and HH 204 (marked on the $\mathrm{H} \alpha$ velocity image in Fig. 28, top panel). A few more small-scale features are visible just south of the Trapezium stars, using the coordinatebased designation system for M42 as invented by O'Dell \& Wen (1994). The Bright Bar is visible as a region of slightly enhanced velocity; more regions of visibly different redshift are marked with annotated gray ellipses on the $\mathrm{H} \alpha$ velocity map in Fig. 28. The two higher velocity $\left(17 \lesssim v_{\mathrm{H} \alpha} \lesssim 21 \mathrm{~km} \mathrm{~s}^{-1}\right)$ regions were discussed by García-Díaz \& Henney (2007); they were named Red Bay for the region east of the Trapezium, and Red Fan for the part just north of the western end of the Bright Bar. The elongated structure of lower velocities between Red Bay, Bright Bar, and Red Fan was discussed in Doi et al. (2004) and named Big Arc with a less pronounced eastern component (11 $\lesssim v_{\mathrm{H} \alpha} \lesssim 14 \mathrm{~km} \mathrm{~s}^{-1}$ in our data) and a stronger and longer southern part ( $3 \lesssim v_{\mathrm{H} \alpha} \lesssim 14 \mathrm{~km} \mathrm{~s}^{-1}$ in the MUSE data). This map 


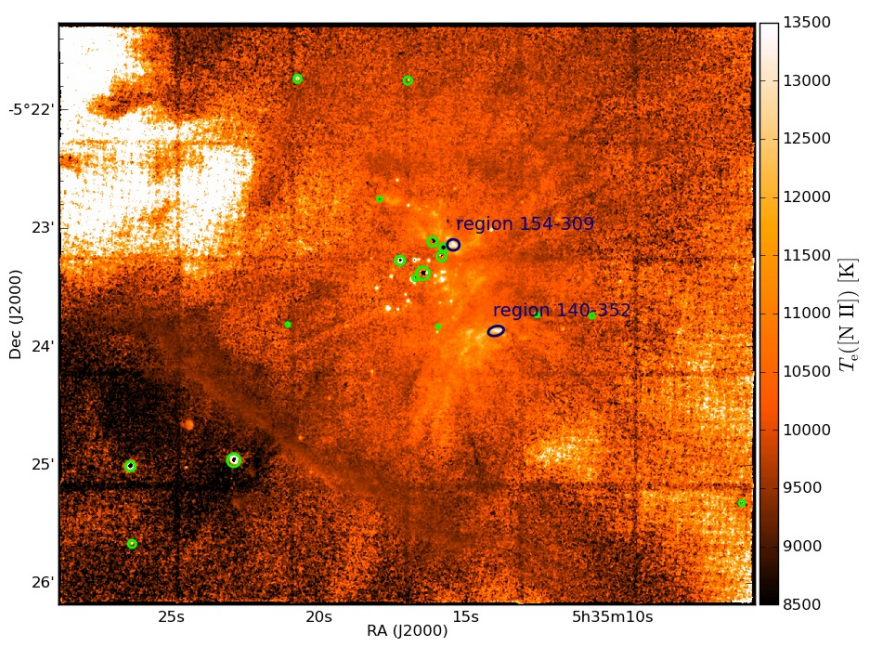

Fig. 24. [N II]-derived $T_{\mathrm{e}}$-map of the central Orion Nebula, smoothed by a median filter of $5 \times 5$ pixels box width, displayed in linear scaling. See text for more details.

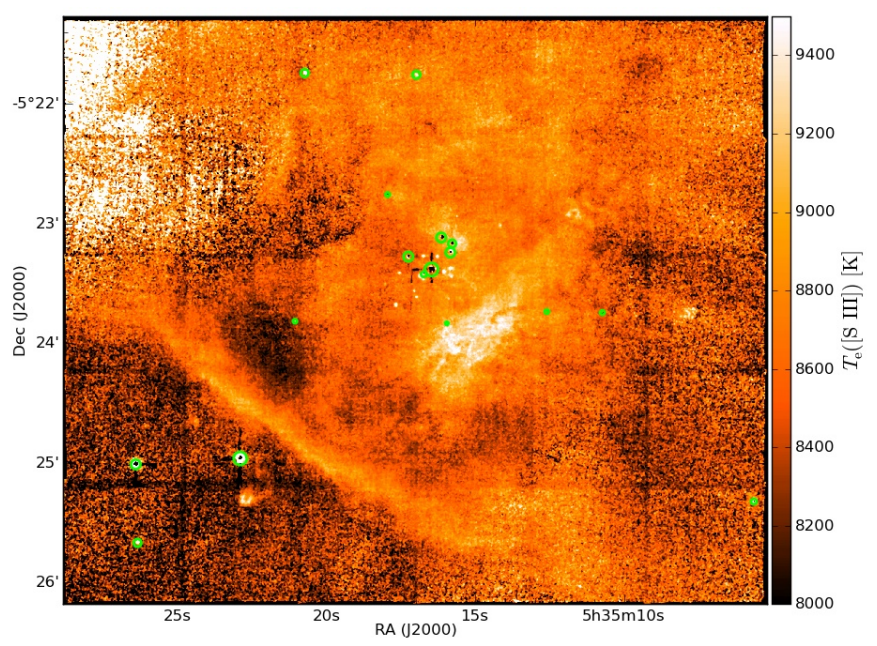

Fig. 25. [S III]-derived $T_{\mathrm{e}}$-map of the central Orion Nebula, smoothed by a median filter of $5 \times 5$ pixels box width, displayed in linear scaling. See text for more details.

can be compared to the $\mathrm{H} \alpha$ velocity map shown by García-Díaz et al. (2008, their Fig. 13). In both datasets, the large scale features show up in a very similar way. Even smaller features, like the velocity dips south of the Trapezium (especially at 168-358 and 161-354) are visibly similar. The absolute velocity values are slightly different between our data and theirs, with their value $\sim 2.5 \mathrm{~km} \mathrm{~s}^{-1}$ higher. This difference is within the combined error estimates of both datasets ${ }^{15}$.

To highlight differences in velocity derived from our data for different ions, we also show the velocities derived for [N II] 6584, subtracted from the $\mathrm{H} \alpha$ velocity field (Fig. 28, middle panel). The velocity offset shown in this map is around $-3.5 \pm 4.8 \mathrm{~km} \mathrm{~s}^{-1}$. Prominent features in this map are HH 202 to the west of the Trapezium and HH 203 and HH 204 beyond the Bright Bar, but on careful investigation it shows a plethora of other features, like blueshifted compact features not related to prominent Herbig-Haro objects, fainter filamentary

\footnotetext{
${ }^{15}$ We computed our velocity map with respect to $\lambda_{\mathrm{H} \alpha}=6562.791 \AA$, see Clegg et al. (1999).
}

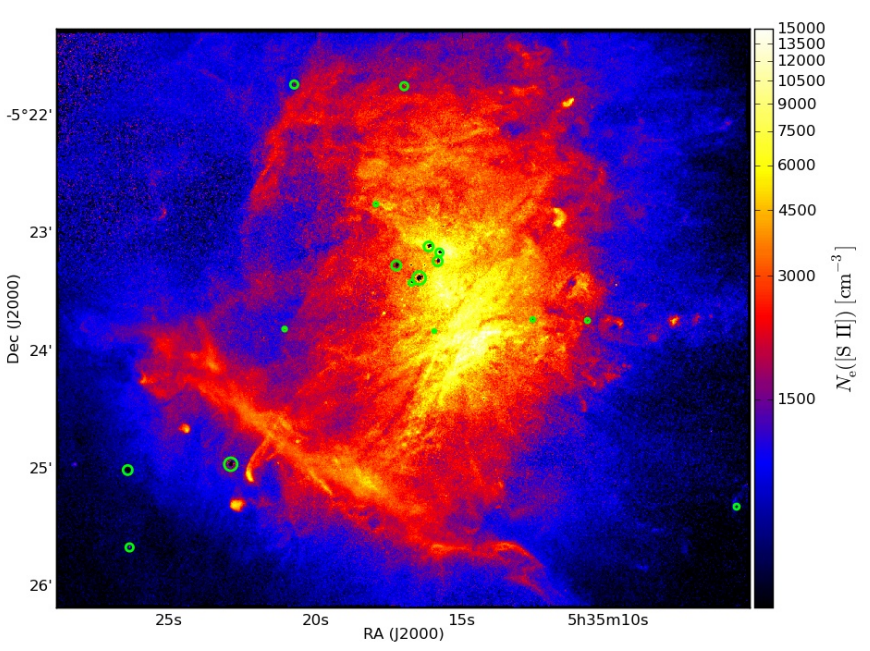

Fig. 26. [S II]-derived $N_{\mathrm{e}}$-map of the central Orion Nebula, smoothed by a median filter of $3 \times 3$ pixels box width, displayed in asinh scaling. See text for more details.

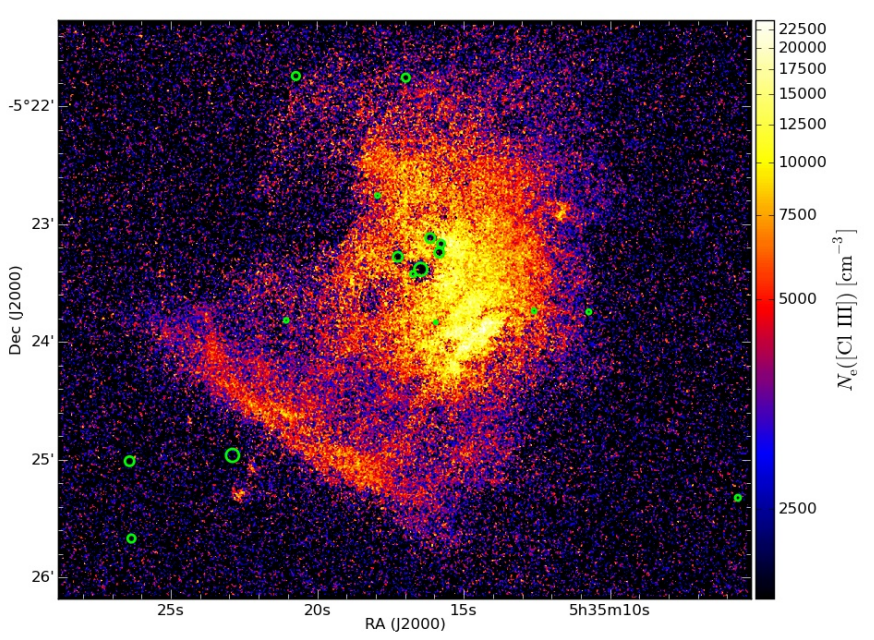

Fig. 27. [Cl III]-derived $N_{\mathrm{e}}$-map of the central Orion Nebula, smoothed by a median filter of $5 \times 5$ pixels box width, displayed in asinh scaling. See text for more details.

structures around the Orion-S region, and smoother large-scale changes. The Big Arc again shows up prominently as a blue region. Constructing the same velocity difference map from the data of García-Díaz et al. (2008) shows a very close match (W. Henney, priv. comm.). Their average velocity difference is $-3.7 \mathrm{~km} \mathrm{~s}^{-1}$ (their Table 2), very close to the value computed here, even though the area covered by their data is not identical.

The bottom panel of Fig. 28 shows the velocity difference map of [S II] 6731 compared to [N II] 6584. The velocity offset between these lines is much smaller, $-2.5 \pm 4.8 \mathrm{~km} \mathrm{~s}^{-1}$. Large-scale features are less pronounced than in the previous velocity maps, and when interpreting them one should keep in mind that the scale shown is far below the velocity resolution achievable with MUSE. Nevertheless, the velocity differences beyond the Bright Bar, i.e., in the region around HH 203 and 204, seem systematically negative. We measure mean values of about $-4 \mathrm{~km} \mathrm{~s}^{-1}$ in that part of the nebula, so that the velocity of the [S II] line is higher in that region than [N II] is, in contrast to the central part where both lines appear to have similar velocities. Another possible velocity difference occurs within the Dark Bay, 

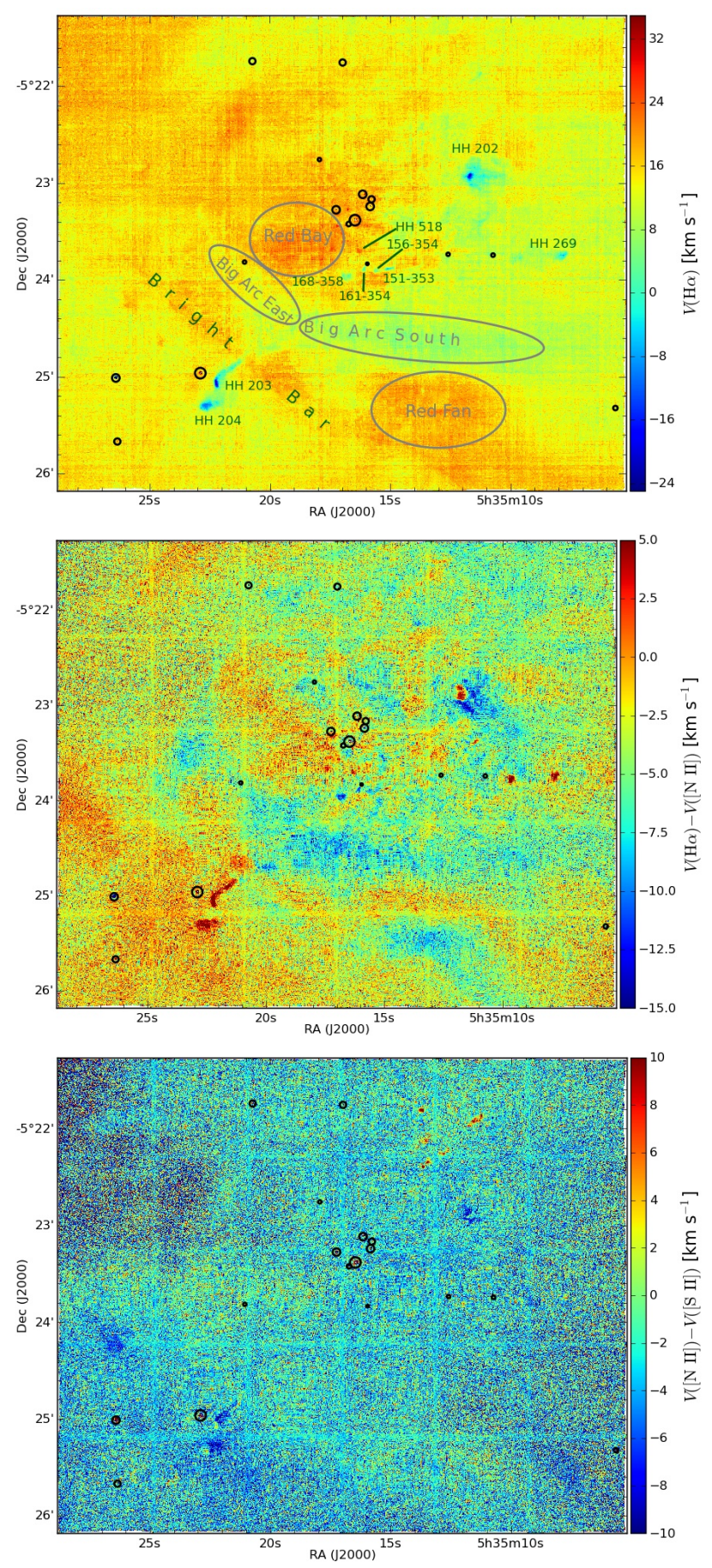

Fig. 28. Velocity field as traced by the lines $\mathrm{H} \alpha$ (top, with respect to barycentric velocity), [N II] 6584 relative to $\mathrm{H} \alpha$ (middle), and [S II] 6731 relative to [N II] 6584 (bottom). We note that the linear color scales are different for all these plots and that the range of the middle plot is asymmetric. The locations of the brighter stars in the field are marked as black circles, further noticeable features are annotated or marked in green or gray on the $\mathrm{H} \alpha$ velocity map.

but the noise in that region is too large to be certain ${ }^{16}$. This map can again be compared to the measurements of García-Díaz et al. (2008). Although their area is slightly smaller, they also find

\footnotetext{
${ }^{16}$ Both in this bottom panel and in the middle panel discussed above, the grid-like structure of the original pointings is visible. This is due to higher $\mathrm{S} / \mathrm{N}$ in the small regions of overlap between. When smoothed spatially, these structures disappear. The velocities measured within and just outside these overlap regions agree well within the error bars.
}

a comparable mean velocity difference of $-0.7 \mathrm{~km} \mathrm{~s}^{-1}$. In the velocity difference map created from their data, one can also determine values of zero in the Red Bay and the Red Fan, like in the MUSE data, but a slightly smaller difference beyond the Bright $\operatorname{Bar}\left(-1.3 \mathrm{~km} \mathrm{~s}^{-1}\right.$, W. Henney, priv. comm.). Small-scale features are again the well-known jets of HH 203 and HH 204, as well as $\mathrm{HH} 202$. Here, some of the outflows from Orion-BN/KL as recently observed in the near-infrared by Bally et al. (2015) show up prominently as red patches to the northwest of the Trapezium, especially НH 201, НH 209, and features near HН 208.

\section{Conclusions}

We successfully demonstrated the capabilities of the integral field spectrograph MUSE instrument with a new dataset representing imaging spectroscopy of the Huygens region of the Orion Nebula. The cubes we provide are among the largest 3D spectroscopic mosaics created so far. We showed that the MUSE data are of high quality in terms of positional accuracy, atmospheric refraction correction, $V$-band magnitude reconstruction, velocities, and flux calibration. We also pointed out artifacts in and imperfections in the data, and explained why two representations in cube form are necessary to cover all possible investigations.

The ensemble of data presented here already allows a variety of science topics to be investigated. In this paper, we restricted our analysis and gave only a demonstration with a simple analysis of the ionized gas in the nebula. We derived the extinction towards the ionization front, and the electron temperature and density using two different emission line ratios, showing the physical properties in different layers of the warm gas around $\theta^{1}$ Ori $C$ and the Trapezium cluster. Further analysis of this data will be presented in Mc Leod et al. (2015) where we will analyze structures and kinematics in the Orion Nebula.

We note that smart spatial binning of the existing MUSE data would enable the detection of weaker spectral features that in our pixel-by-pixel analysis are detected with sufficient $\mathrm{S} / \mathrm{N}$ only in the central part. In the long term it might prove useful to reobserve the field with MUSE, with possibly a longer exposure time and/or more exposures per pointing, perhaps mapping an even larger field. This would allow physical properties to be estimated from fainter emission lines, and properties to be mapped with even lower systematic effects to the outskirts of M 42.

Until that time, the current dataset is of high quality and already maps the most interesting area of the Orion Nebula in the optical wavelength range. It can serve as a reference for many follow-up studies. To enable the widest possible use of these exceptional legacy data for science questions from members of the community, we publicly release the complete reduced cubes, as well as the inferred reddening, density, and temperature maps ${ }^{17}$.

Acknowledgements. The authors thank the (rest of) the MUSE consortium, the teams that conducted first light and commissioning observations, and support from ESO during these activities, as well as S. Conseil and the MUSE Data Management Team for setting up webpage and data storage. We also thank C. R. O'Dell and G. Ferland for helpful comments. We thank our referee, W. Henney for an insightful and detailed report that enabled us to improve several critical points. PMW and SK received funding through BMBF Verbundforschung (project MUSE-AO, grant 05A14BAC and 05A14MGA). AMI acknowledges support from Agence Nationale de la Recherche through the STILISM project (ANR-12-BS05-0016-02). RB acknowledges support from the ERC advanced grant 339659-MUSICOS. We are grateful to the developers of software such as SAOImage DS9 (developed by Smithsonian Astrophysical Observatory, http: //ds9.si.edu/), IRAF, PyNeb, APLpy (http://aplpy.github.com), and topcat (http: //www.starlink.ac.uk/topcat/), without which work on this

17 http://muse-vlt.eu/science 
data would have been much more difficult. We also thank the AstroPy community for useful discussions. This research has made use of the SIMBAD database, operated at CDS, Strasbourg, France.

\section{References}

Alonso-Herrero, A., García-Marín, M., Monreal-Ibero, A., et al. 2009, A\&A, 506,1541

Arthur, S. J., \& Hoare, M. G. 2006, ApJS, 165, 283

Bacon, R., Vernet, J., Borisova, E., et al. 2014, The Messenger, 157, 13

Baldwin, J. A., Ferland, G. J., Martin, P. G., et al. 1991, ApJ, 374, 580

Baldwin, J. A., Verner, E. M., Verner, D. A., et al. 2000, ApJS, 129, 229

Bally, J., O'Dell, C. R., \& McCaughrean, M. J. 2000, AJ, 119, 2919

Bally, J., Ginsburg, A., Silvia, D., \& Youngblood, A. 2015, A\&A, 579, A130

Blagrave, K. P. M., Martin, P. G., Rubin, R. H., et al. 2007, ApJ, 655, 299

Bohlin, R. C., Savage, B. D., \& Drake, J. F. 1978, ApJ, 224, 132

Cairós, L., Caon, N., \& Weilbacher, P. 2015, A\&A, 577, A21

Cardelli, J. A., Clayton, G. C., \& Mathis, J. S. 1989, ApJ, 345, 245

Clegg, R. E. S., Miller, S., Storey, P. J., \& Kisielius, R. 1999, A\&AS, 135, 359

Da Rio, N., Robberto, M., Soderblom, D. R., et al. 2009, ApJS, 183, 261

De Robertis, M. M., Dufour, R. J., \& Hunt, R. W. 1987, J. Roy. Astron. Canada, 81,195

Doi, T., O’Dell, C. R., \& Hartigan, P. 2004, AJ, 127, 3456

Doi, M., Tanaka, M., Fukugita, M., et al. 2010, AJ, 139, 1628

Ducati, J. R. 2002, VizieR Online Data Catalog: II/237

Filippenko, A. V. 1982, PASP, 94, 715

Foukal, P. 1974, PASP, 86, 211

García-Díaz, M. T., \& Henney, W. J. 2007, AJ, 133, 952

García-Díaz, M. T., Henney, W. J., López, J. A., \& Doi, T. 2008, Rev. Mex. Astron. Astrofis., 44, 181

García-Marín, M., Colina, L., Arribas, S., \& Monreal-Ibero, A. 2009, A\&A, 505, 1319

Graham, M. F., Meaburn, J., \& Redman, M. P. 2003, MNRAS, 343, 419

Hillenbrand, L. A. 1997, AJ, 113, 1733

Israel, F. P. 1978, A\&A, 70, 769

Johnstone, D., \& Bally, J. 1999, ApJ, 510, L49

Johnstone, D., \& Bally, J. 2006, ApJ, 653, 383

Kamann, S., Wisotzki, L., \& Roth, M. M. 2013, A\&A, 549, A71

Kelz, A., Verheijen, M. A. W., Roth, M. M., et al. 2006, PASP, 118, 129

Kramida, A., Yu. Ralchenko, Reader, J., \& NIST ASD Team 2014, NIST Atomic Spectra Database (v5.2) [http://physics.nist.gov/asd]

Luridiana, V., Morisset, C., \& Shaw, R. A. 2013, PyNeb: Analysis of emission lines, Astrophysics Source Code Library [record ascl : 1304.021]

Luridiana, V., Morisset, C., \& Shaw, R. A. 2015, A\&A, 573, A42

Mc Leod, A. F., Weilbacher, P. M., Ginsburg, A., et al. 2015, MNRAS, submitted

Mesa-Delgado, A., Esteban, C., \& García-Rojas, J. 2008, ApJ, 675, 389

Mesa-Delgado, A., Núñez-Díaz, M., Esteban, C., López-Martín, L., \& García-Rojas, J. 2011, MNRAS, 417, 420
Mesa-Delgado, A., Núñez Díaz, M., Esteban, C., et al. 2012, MNRAS, 426, 614

Noll, S., Kausch, W., Kimeswenger, S., et al. 2014a, A\&A, 567, A25

Noll, S., Kausch, W., Kimeswenger, S., et al. 2014b, Skycorr: Sky emission subtraction for observations without plain sky information, Astrophysics Source Code Library

Núñez-Díaz, M., Mesa-Delgado, A., Esteban, C., et al. 2012, MNRAS, 421, 3399

Núñez-Díaz, M., Esteban, C., \& Mesa-Delgado, A. 2013, in Highlights of Spanish Astrophysics VII, eds. J. C. Guirado, L. M. Lara, V. Quilis, \& J. Gorgas, 594

O’Dell, C. R. 2001, ARA\&A, 39, 99

O'Dell, C. R., \& Harris, J. A. 2010, AJ, 140, 985

O'Dell, C. R., \& Henney, W. J. 2008, AJ, 136, 1566

O’Dell, C. R., \& Wen, Z. 1994, ApJ, 436, 194

O'Dell, C. R., \& Yusef-Zadeh, F. 2000, AJ, 120, 382

O’Dell, C. R., Henney, W. J., Abel, N. P., Ferland, G. J., \& Arthur, S. J. 2009, AJ, 137, 367

Osterbrock, D. E., Tran, H. D., \& Veilleux, S. 1992, ApJ, 389, 305

Osterbrock, D. E., \& Ferland, G. J. 2005, Astrophysics of gaseous nebulae and active galactic nuclei, 2nd. edn. (University Science Books)

Patat, F., Moehler, S., O’Brien, K., et al. 2011, A\&A, 527, A91

Pogge, R. W., Owen, J. M., \& Atwood, B. 1992, ApJ, 399, 147

Robberto, M., Soderblom, D. R., Bergeron, E., et al. 2013, ApJS, 207, 10

Rosado, M., de la Fuente, E., Arias, L., Raga, A., \& Le Coarer, E. 2001, AJ, 122, 1928

Samus, N. N., Durlevich, O. V., et al. 2009, VizieR Online Data Catalog, I/2025

Sánchez, S. F., Cardiel, N., Verheijen, M. A. W., et al. 2007, A\&A, 465, 207

Shields, G. 1990, ARA\&A, 28, 525

Skrutskie, M. F., Cutri, R. M., Stiening, R., et al. 2006, AJ, 131, 1163

Smette, A., Kausch, W., Sana, H., et al. 2015a, Molecfit: Telluric absorption correction tool, Astrophysics Source Code Library

Smette, A., Sana, H., Noll, S., et al. 2015b, A\&A, 576, A77

Storey, P. J., \& Hummer, D. G. 1995, MNRAS, 272, 41

Strömgren, B. 1939, ApJ, 89, 526

Takahashi, S., Ho, P. T. P., Teixeira, P. S., Zapata, L. A., \& Su, Y.-N. 2013, ApJ, 763,57

Takami, M., Usuda, T., Sugai, H., et al. 2002, ApJ, 566, 910

Tsamis, Y. G., \& Walsh, J. R. 2011, MNRAS, 417, 2072

Tsamis, Y. G., Flores-Fajardo, N., Henney, W. J., Walsh, J. R., \& Mesa-Delgado, A. 2013, MNRAS, 430, 3406

van der Werf, P. P., Goss, W. M., \& O’Dell, C. R. 2013, ApJ, 762, 101

Vasconcelos, M. J., Cerqueira, A. H., Plana, H., Raga, A. C., \& Morisset, C. 2005, AJ, 130, 1707

Weilbacher, P. M., Duc, P.-A., \& Fritze-von Alvensleben, U. 2003, A\&A, 397, 545

Weilbacher, P. M., Streicher, O., Urrutia, T., et al. 2012, in Software and Cyberinfrastructure for Astronomy II, Proc. SPIE, 8451

Yusef-Zadeh, F. 1990, ApJ, 361, L19

Zuckerman, B. 1973, ApJ, 183, 863 